\title{
Statistics of X-ray observables for the cooling-core and non-cooling core galaxy clusters ${ }^{\star}$
}

\author{
Y. Chen ${ }^{1,2}$, T. H. Reiprich ${ }^{3}$, H. Böhringer ${ }^{2}$, Y. Ikebe ${ }^{4}$, and Y.-Y. Zhang ${ }^{2}$ \\ 1 Key Laboratory of Particle Astrophysics, Institute of High Energy Physics, Chinese Academy of Sciences, \\ Beijing 100049, PR China \\ e-mail: ychen@mail.ihep.ac.cn \\ 2 Max-Planck-Institut für Extraterrestrische Physik, 85748 Garching, Germany \\ 3 Argelander-Institut für Astronomie, Universität Bonn, Auf dem Hügel 71, 53121 Bonn, Germany \\ ${ }^{4}$ National Museum of Emerging Science and Innovation, Tokyo, 135-0064, Japan
}

Received 29 September 2006 / Accepted 17 December 2006

\section{ABSTRACT}

\begin{abstract}
We present a statistical study of the occurrence and effects of the cooling cores in the clusters of galaxies in a flux-limited sample, HIFLUGCS, based on ROSAT and ASCA observations. About $49 \%$ of the clusters in this sample have a significant, classicallycalculated cooling-flow, mass-deposition rate. The upper envelope of the derived mass-deposition rate is roughly proportional to the cluster mass, and the fraction of cooling core clusters is found to decrease with it. The cooling core clusters are found to have smaller core radii than non-cooling core clusters, while some non-cooling core clusters have high $\beta$ values $(>0.8)$. In the relation of the $\mathrm{X}$-ray luminosity vs. the temperature and the mass, the cooling core clusters show a significantly higher normalization. A systematic correlation analysis, also involving relations of the gas mass and the total infrared luminosity, indicates that this bias is shown to be mostly due to an enhanced X-ray luminosity for cooling core clusters, while the other parameters, like temperature, mass, and gas mass may be less affected by the occurrence of a cooling core. These results may be explained by at least some of the non-cooling core clusters being in dynamically young states compared with cooling core clusters, and they may turn into cooling core clusters in a later evolutionary stage.
\end{abstract}

Key words. galaxies: clusters: general - galaxies: intergalactic medium - X-ray: galaxies: clusters

\section{Introduction}

Clusters of galaxies are interesting large-scale astrophysical laboratories offering ideal probes for studying the large-scale structure of the Universe and for testing the cosmological models (e.g. Voit 2005). A very important scaling parameter in these studies is the cluster mass, which cannot easily be measured unless detailed observations are available. It is therefore estimated by means of other suitable, easily obtained global observables such as X-ray luminosity or X-ray temperature (e.g. Reiprich \& Böhringer 2002; Markevitch 1998; Ikebe et al. 2002; Finoguenov et al. 2001; Arnaud et al. 2005). Since the early days of X-ray imaging with the EINSTEIN satellite, it is apparent that there may be two, to some extent distinct, classes of galaxy clusters: clusters with very dense gaseous core regions, so-called cooling cores, and another type with shallower cores often exhibiting a more internal structure (e.g. Jones \& Forman 1984; Ota \& Mitsuda 2004; Peres et al. 1998; Schuecker et al. 2001a). In the present paper we explore first the influence of this dichotomy on the scaling relations between global cluster X-ray observables and then between the observables and the cluster mass in order to improve our understanding of how to use these scaling relations in cosmological applications.

Clusters with dense gaseous cores, which have central cooling times significantly lower than a Hubble time, have formerly been termed cooling flow clusters, and it was believed

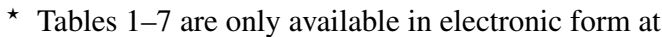
http://www . aanda. org that the intracluster medium (ICM) in these regions cools and condenses, as it is difficult to avoid cooling in the absence of a very fine-tuned heating mechanism (Fabian 1994). A different point of view not requiring a cooling flow has also been put forward based on ASCA spectroscopic results (e.g., Ikebe et al. 1999; Makishima et al. 2001, and references therein). With XMM-Newton observations, it was found that the spectral features predicted by the classical cooling flow model are not observed in the X-ray spectra of cooling flow regions (e.g. Peterson et al. 2001, 2003). While a slight temperature decrease by factors up to 2-3 towards the center in cooling cores is observed, the expected features for further cooling are absent. With high-resolution Chandra observations, a possible fine-tuned heat source has been found in the interaction of central AGN with the cluster ICM, which is now taken as the most probable reason for the prevention of massive cooling flows (e.g. David et al. 2001; Böhringer et al. 2002; Fabian et al. 2003; McNamara et al. 2005). Therefore we follow the now widely-used convention to call the clusters in our sample cooling core clusters (CCC) and non-cooling core clusters (NCCC).

An influence of the CCC or NCCC nature of the clusters on the scaling relations of global properties has previously been realized, e.g., in the luminosity temperature relation (e.g. Fabian et al. 1994; Markevitch 1998; McCarthy et al. 2004) and other parameters (O'Hara et al. 2006). Here we extend the studies of the influence of CCCs on the scaling relation to the largest X-ray flux limited sample of galaxy cluster with detailed X-ray data that allow a mass determination, the HIFLUGCS (the HIghest 
X-ray FLUx Galaxy Cluster Sample; Reiprich 2001; Reiprich \& Böhringer 2002). This cluster sample is selected only by X-ray flux, irrespective of the cluster morphology, and we do not know of any signature of incompleteness in the sample. Therefore it should provide a representative mix of cluster morphologies for a given X-ray luminosity, providing the correct statistics be applied to the typical cosmological X-ray survey cluster samples.

In particular we study the segregation of $\mathrm{CC}$ and NCC clusters in the $L_{\mathrm{X}}-T, L_{\mathrm{X}}-M, M-T$, and the $f_{\mathrm{gas}}-T$ relations. A major goal in this study is to better understand the scatter in these relations, which has to be folded into the test of large-scale structure measures and cosmological models (e.g., Ikebe et al. 2002; Stanek et al. 2006). It is especially interesting in the context of the $L_{X}-M$ relation given by Reiprich \& Böhringer (2002) where the observed scatter is very large and partly due to the large uncertainties in mass determination. Therefore it was very difficult to separate the intrinsic scatter from the scatter introduced by the formal and systematic measurement errors. It is the intrinsic scatter, however, that is important for the application. A difference in the relation amplitude between CCCs and NCCCs could in principle provide a lower limit to the intrinsic scatter in the $L_{X}-M$ relation of Reiprich \& Böhringer (2002), if the systematic uncertainties are well controlled, and thus help to understand the origin of the scatter better. This is interesting because the best-fit cosmological parameter values from the WMAP 3rd year data (Spergel et al. 2006) applied to compare the predicted and observationally derived HIFLUGCS cluster mass function provide an indication that the intrinsic scatter is probably smaller than the systematic measurement errors that go into the derived mass and X-ray luminosity relation (Reiprich 2006). Our study has a lot in common with the work of O'Hara et al. (2006), but was started independently a few years ago, so we discuss the correspondence of the two studies throughout the paper.

The paper is organized as follows. In Sect. 2 we briefly introduce the sample. In Sect. 3, we present the method of data reduction. We compare the properties of the CCC and NCCC in Sect. 4 and discuss the implications of the results. Section 5 provides a summary. In the following we adopt a cosmological model with $H_{0}=50 \mathrm{~km} \mathrm{~s}^{-1} \mathrm{Mpc}^{-1}, \Omega_{\mathrm{m}}=1$ and $\Omega_{\Lambda}=0$, a choice which was mostly made for easier comparison with previous results.

\section{The sample}

The extended HIFLUGCS sample with 106 clusters and groups of galaxies is used for the present study. Ninety-two of these clusters have known temperatures determined from X-ray spectroscopy. Here we use two cluster temperatures: $T_{\mathrm{m}}$, the emission measure weighted temperature, which is mainly derived from a single temperature fit to the global X-ray spectrum of the clusters (Markevitch et al. 1998; Reiprich 2001, and references therein); and $T_{\mathrm{h}}$, which is the hotter bulk component of a two-temperature model fitted to the spectrum ( 88 of them from ASCA, Ikebe et al. 2002). The $T_{\mathrm{h}}$ was determined by accepting a small, second lower-temperature component, to allow for a low temperature phase in a possible cooling core in the central cluster region. The typical temperature of this second component was about a factor of 2 lower than the bulk temperature (Ikebe et al. 2002). The second component of the two-temperature fit, which is generally only needed for the cooling core clusters, has a small normalization and is expected to account for the lower central temperature phase in the cooling cores. For the clusters with no measured $T_{\mathrm{m}}$ or $T_{\mathrm{h}}$, we derived them using the $L_{\mathrm{X}}-T$ relation of Markevitch (1998) with the $L_{X}(<2 \mathrm{Mpc})$ uncorrected

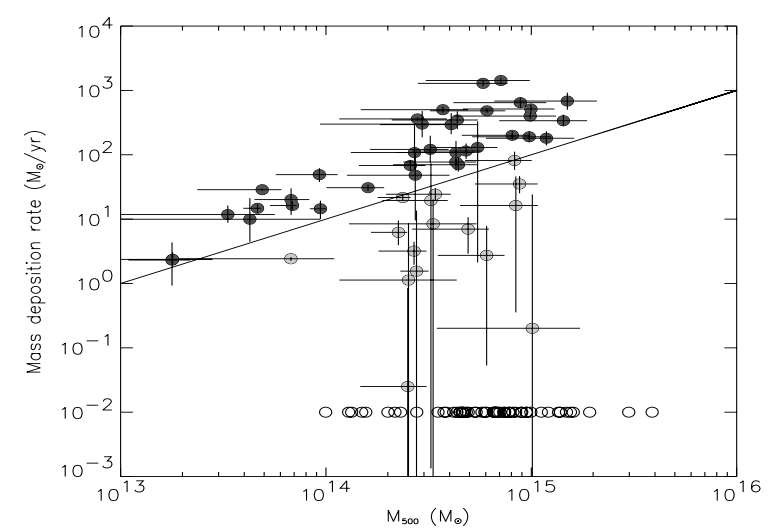

Fig. 1. Formally-deduced mass-deposition rates in the frame of the classical cooling flow model as a function of the total cluster mass, $M_{500}$. There is a pronounced ridge line of stronger cooling core clusters with a formal mass deposition rate almost proportional to the cluster mass. The clusters with insignificant mass deposition rates below a value of $0.01 M_{\odot} \mathrm{yr}^{-1}$ are plotted according to their total mass at the bottom of the plot with a formal value of $0.01 M_{\odot} \mathrm{yr}^{-1}$. The line at $\dot{M} / M_{500}=10^{-13} \mathrm{yr}^{-1}$ separates strong CCCs from small-to-moderate CCCs. In all figures of this paper, the filled black circles represent the pronounced CCCs. The filled grey circles are the small-to-moderate CCCs and the open circles are NCCCs. Throughout this paper the error bars are at a $\pm 68 \%$ confidence level except for $T_{\mathrm{m}}$ of some clusters, $T_{\mathrm{h}}$, and $L_{\mathrm{X}}$, which are at a $90 \%$ confidence level (and thus a conservative error estimate).

and $T$ corrected for cooling flows. The hotter component, $T_{\mathrm{h}}$, which is usually slightly higher than $T_{\mathrm{m}}$, is expected to provide a good measure of the gravitational potential depth and the total mass of the clusters.

The X-ray surface brightness profiles are derived from ROSAT PSPC observations, 36 of them are from RASS observations (allowing for a large enough field-of-view for the prominent nearby clusters), and 70 from pointing observations (Reiprich \& Böhringer 2002). The large FOV of the ROSAT PSPC allows us to cover most of the clusters out to $r_{500}$, the radius at which the mean density of the cluster is 500 times that of a critical density universe. In addition, it is worth noting that this flux limited sample has the largest sky area so far.

The basic properties of the clusters in the HIFLUGCS sample from Reiprich \& Böhringer (2002) are given in Tables 1 and 2 . Note that $T_{\mathrm{m}}$, the emission-measure weighted temperature, is used in the calculation of some quantities, such as $n_{\text {center }}$, $t_{\text {cool }}$, and $\dot{M}$ in the next section. In Fig. 1 we show the distribution of the masses and cooling flow mass deposition rates determined as described in the following sections. The NCC clusters with no significant mass-deposition rates and with very small or no cooling radii are shown with their mass distribution at the bottom of the plot. A striking feature of the plot is the ridge of cooling core clusters with formal mass deposition rates that increase almost linearly with cluster mass. This leads us to define the class of pronounced cooling core clusters by a lower limit to the ratio of the formal mass deposition rate to the cluster mass, $M_{500}$. The ratio value chosen for $\dot{M} / M_{500}$ of $10^{-13} \mathrm{yr}^{-1}$ is indicated in Fig. 1 . A further limit in the mass deposition rate at $0.01 M_{\odot} \mathrm{yr}^{-1}$ is used to separate small-to-moderate cooling cores from NCCC. The total sample thus splits up into 36 pronounced CCCs, 16 smallto-moderate CCCs, and 54 NCCCs. We make use of this classification below. 


\section{Data analysis}

\subsection{X-ray surface brightness and mass profiles}

To determine the X-ray surface brightness distribution we produced images in the 0.5 to $2 \mathrm{keV}$ band (PSPC PI channel 52 to 201) and the corresponding vignetting-corrected exposure maps. The contaminating point sources and obvious substructure were excised. A center position was obtained from an iterative determination of the "center-of-mass" of the photon distribution in a 7.5 arcmin radius aperture. The surface brightness profile was then constructed by azimuthal averaging in concentric bins. This procedure is the same as used in Reiprich \& Böhringer (2002).

We fit the surface brightness profile with a single $\beta$ model (Cavaliere \& Fusco-Femiano 1976)

$S(r)=S_{0}\left(1+\left(r / r_{\mathrm{c}}\right)^{2}\right)^{-3 \beta+1 / 2}$,

where $S_{0}$ is central brightness (counts/s/pixel ${ }^{2} ; 1^{\prime}=120$ pixels for ROSAT PSPC) and $r_{\mathrm{c}}$ the core radius (kpc). We also try a double $\beta$ model

$S(r)=S_{01} \alpha_{1}^{-3 \beta_{1}+1 / 2}+S_{02} \alpha_{2}^{-3 \beta_{2}+1 / 2}$,

$\alpha_{1}=1+\left(r / r_{\mathrm{c} 1}\right)^{2}$,

$\alpha_{2}=1+\left(r / r_{\mathrm{c} 2}\right)^{2}$,

in which the fits of 49 clusters have significantly improved reduced $\chi^{2}$ values compared to the fits using a single $\beta$ model (Table 3). We thus use the double $\beta$ model for these clusters. Assuming that the temperature is homogeneous in the cluster, we can calculate the gas number-density profile $n(r)$. The errors introduced by this simplification in the presence of temperature variations is only on the order a few percent, which justifies this approximation. Assuming, moreover, that the gas is in hydrostatic equilibrium, the total mass of the cluster can be calculated for a single $\beta$ model as

$M(r)=\frac{3 \beta k T_{\mathrm{h}} r}{G \mu m_{\mathrm{p}}} \frac{\left(r / r_{\mathrm{c}}\right)^{2}}{1+\left(r / r_{\mathrm{c}}\right)^{2}}$,

where $k$ is the Boltzmann constant, $G$ the gravitational constant, $\mu$ the molecular weight $(\mu=0.61)$, and $m_{\mathrm{p}}$ the proton mass. For a double $\beta$ model we find

$M(r)=\frac{3 k T_{\mathrm{h}} r^{3}}{G \mu m_{\mathrm{p}}} \frac{n_{01}^{2} \beta_{1} \alpha_{1}^{-3 \beta_{1}-1} / r_{\mathrm{c} 1}^{2}+n_{02}^{2} \beta_{2} \alpha_{2}^{-3 \beta_{2}-1} / r_{\mathrm{c} 2}^{2}}{n_{01}^{2} \alpha_{1}^{-3 \beta_{1}}+n_{02}^{2} \alpha_{2}^{-3 \beta_{2}}}$,

where $n_{01}$ and $n_{02}$ are the central equivalent electron number density calculated from the two surface brightness components. The central electron number density, $n_{0}$, can be derived from

$n_{0}^{2}=n_{01}^{2}+n_{02}^{2}$.

\subsection{Cooling core properties}

The cooling time of the gas is calculated by

$t_{\text {cool }}=\frac{5}{2} \frac{n_{\mathrm{e}}+n_{\mathrm{i}}}{n_{\mathrm{e}}} \frac{k T_{\mathrm{m}}}{n_{\mathrm{H}} \Lambda\left(A, T_{\mathrm{m}}\right)}$,

where $\Lambda\left(A, T_{\mathrm{m}}\right)$ is the cooling function of the gas, and $n_{\mathrm{e}}, n_{\mathrm{i}}$, and $n_{\mathrm{H}}$ are the number densities of the electrons, ions, and hydrogen, respectively. Here we use the abundance $A=0.3$ for all clusters. Note that for the nearly fully ionized plasma in clusters, $n_{\mathrm{e}}=1.2 n_{\mathrm{H}}$ and $n_{\mathrm{i}}=1.1 n_{\mathrm{H}}$.

Following the previously most frequently-used convention, we define the cooling radius as the radius where the gas cooling time is equal to the age of the cluster assumed to be close to the Hubble time ( $\left.t_{\text {age }} \sim 1 / H_{0}=13 \mathrm{Gyr}\right)$. The physical meaning of the cooling radius within the classical cooling flow model is that, within the cooling radius, the gas will lose all of its energy by $\mathrm{X}$-ray emission and is replaced by ambient hot gas from larger radii in a steady state inflow. We can therefore calculate the energy loss rate from the integral of the X-ray emission inside the cooling radius and the mass inflow rate from the enthalpy influx necessary to compensate for this energy loss. We then account for the energy gain as the inflowing gas moves down the gravitational potential gradient. Thus, the total mass deposition rate within the shell $i$ can be determined by

$\dot{M}(i)=\frac{n_{\mathrm{e}}(i) n_{\mathrm{H}}(i) \Lambda\left(A, T_{\mathrm{m}}\right) V(i)+\frac{5}{2} \frac{k T_{\mathrm{m}}}{\mu m_{\mathrm{p}}} \dot{M}(i-1)}{\frac{5}{2} \frac{k T_{\mathrm{m}}}{\mu m_{\mathrm{p}}}+\Phi(i+1)-\Phi(i)}$,

where $V(i)$ is volume of the shell $i$, and $\Phi(i+1)$ and $\Phi(i)$ are the gravitational potential of the shell $i+1$ and $i$, respectively. For a single $\beta$ model, $\Phi(i)$ can be calculated from

$\Phi(i)=\frac{3}{2} \frac{\beta k T_{\mathrm{h}}}{\mu m_{\mathrm{p}}} \ln \left(1+\left(\frac{r(i)}{r_{\mathrm{c}}}\right)^{2}\right)$.

When we use the double $\beta$ model, these formulae will change accordingly, and we limit their writing-out for brevity.

\subsection{Error estimate}

We adopted a Monte-Carlo method to estimate the errors of the cluster properties derived in the previous subsection, such as the mass and mass deposition rate. In the calculation of the mass of the clusters, we assumed a polytropic index with a value of $\gamma=$ 1. From previous observational studies, the range of $\gamma$-values is constrained to be between 0.9 and 1.3 (e.g. Finoguenov 2001; Pratt et al. 2006). The main errors are from the temperature and the $\gamma$. We used a $\beta \gamma$ model (e.g. Ettori 2000) to estimate the errors of the mass deposition rate and assumed a polytropic index greater than 0.9 and following a Gaussian distribution with a mean value of 1.15 and a variance of 0.15 as derived in Finoguenov et al. (2001). For each cluster in the HIFLUGCS, we created a cluster sample with 1000 clusters with simulated $T_{\mathrm{m}}$, $T_{\mathrm{h}}, S, \beta, r_{\mathrm{c}}$, and $\gamma$ according to their own errors. We calculated other properties (e.g. $n, t_{\text {cool }}, \dot{M}, M$ and so on) of each simulated cluster and then obtained the errors.

\section{Statistical properties}

In the following we investigate the relations between several observables and the cluster mass. For all the relations we use the BCES-Bisector fit of Akritas \& Bershady (1996). The fits are performed with the logarithmic values of the parameters and quoted in the form

$\log _{10}(Y)=A+B \cdot \log _{10}(X)$

in Table 7. The median X-ray luminosity, $L_{X}(0.1-2.4 \mathrm{keV})$, the median temperature, $T_{\mathrm{m}}$, and the median cluster mass, $M_{500}$ of the sample are $2.9 \times 10^{44} \mathrm{erg} \mathrm{s}^{-1}, 4.1 \mathrm{keV}$, and $4.8 \times 10^{14} M_{\odot}$, respectively. Therefore we use the values $1 \times 10^{44} \mathrm{erg} \mathrm{s}^{-1}, 4 \mathrm{keV}$, and $5 \times 10^{14} M_{\odot}$, respectively, as pivot points for the fits of the relations. 


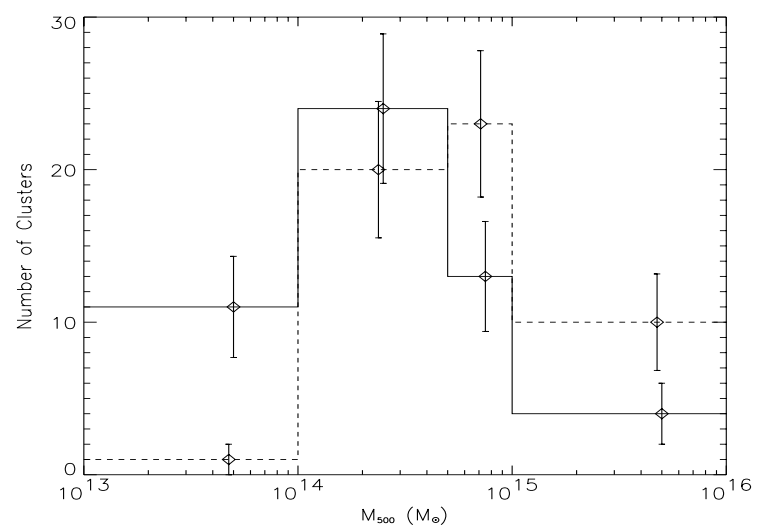

Fig. 2. The numbers of CCCs (solid lines) and NCCCs (dashed lines) versus the mass of the clusters $M_{500}$. Note that the fraction of NCCCs increases with $M_{500}$.

\subsection{Basic properties}

In Fig. 2 we show the number of CCCs (here including the strong CCC and moderate CCC) and NCCCs in the sample as a function of the cluster mass. The fraction of NCCCs clearly increases with $M_{500}$. This is also seen in the smaller flux-limited sample used by O'Hara et al. (2006). At the low $M_{500}$ end, this may partly be due to some small mass non-cooling core groups possibly having low luminosities and not reaching the flux limit of HIFLUGCS. In general, however, the main reasons may be that the fraction of dynamically young clusters increases with cluster mass and that these clusters do not generally feature cooling cores. In addition the ICM is hotter in more massive clusters making the radiative heat loss relatively slower. This is an important statistical property of the cluster sample to keep in mind, since any segregation of CCCs and NCCCs in the parameter relations can then also introduce a mass-dependent effect in the relations of the combined sample.

Figure 3 shows the distribution of the values for the core radius, $r_{\mathrm{c}}$, and slope parameter $\beta$ from the fit of the $\beta$-model to the $\mathrm{X}$-ray surface-brightness profiles of the clusters in the sample. As in previous work (e.g. Jones \& Forman 1984; White et al. 2000; Ota \& Mitsuda 2004), the CC clusters segregate very distinctly at lower values of the core radius than the NCC clusters. Furthermore, we find that the high the relative $\dot{M}_{r}$, the low the $r_{\mathrm{c}}$. In addition, it was found that some NCCCs have high $\beta$ values with $\beta>0.8$, while such high values are not found among the CCCs. There are 2 reasons for this behavior. For similar gravitational potential shapes in CCCs and NCCCs, the $\mathrm{CC}$ clusters with central temperature drop, and a corresponding central ICM density increase in pressure equilibrium feature $\mathrm{X}$-ray surface brightness cusps that are fit by smaller core radii (Jones \& Forman 1984). In addition, NCCCs are often dynamically young, featuring substructure, elongations, or disturbed core regions which result in inflated core radii, that in turn lead to steeper outer surface brightness slopes.

In Fig. 4 we explore the relation of the core radius, $r_{\mathrm{c}}$, with the cluster mass, $M_{500}$. Assuming that clusters have a strictly self-similar shape, we expect that any characteristic radius scales as $r \propto M^{1 / 3}$. The results of the power-law scaling relation fits to the data are given in Table 6 and shown in Fig. 4. For both subsamples, CCCs and NCCCs, the observed slope is steeper than this simple expectation; that is, the core radius increases with mass faster than expected. The explanation for this behavior is probably not trivial. For the NCCCs, the reason might again be that the fraction of dynamically young clusters with inflated core

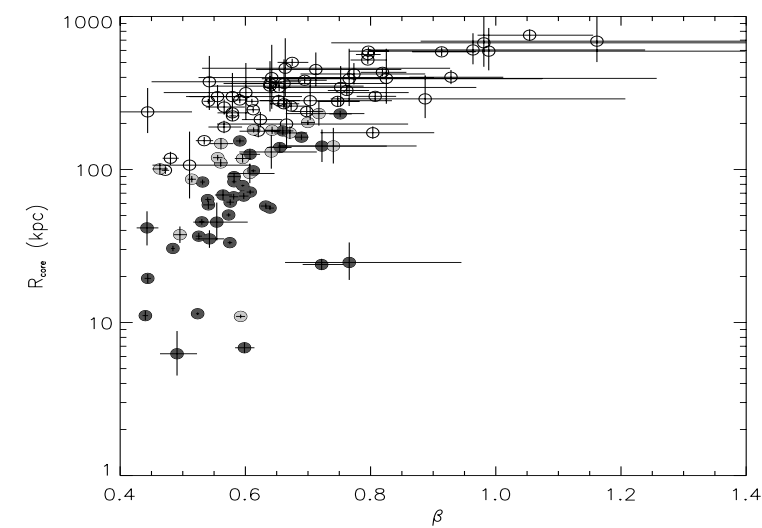

Fig. 3. The $r_{\mathrm{c}}-\beta$ diagram, showing the cooling-core clusters have smaller core radii.

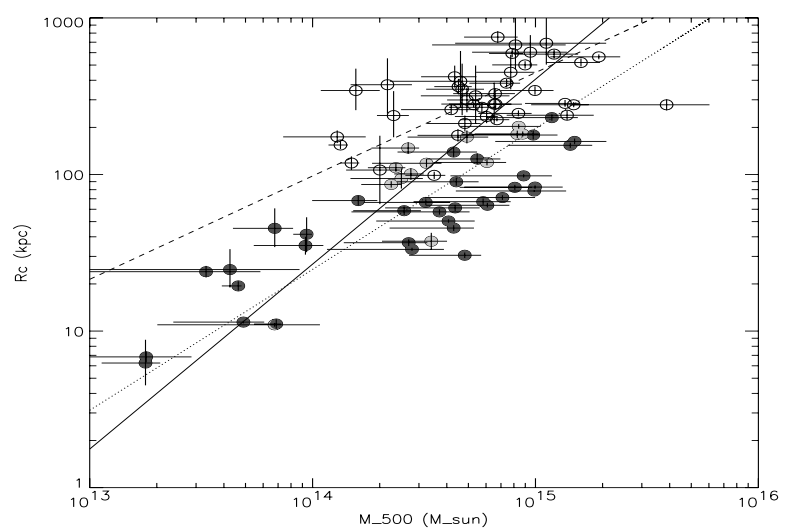

Fig. 4. The $r_{\mathrm{c}}-M_{500}$ diagram. Here $r_{\mathrm{c}}$ increases with $M_{500}$ faster than the self-similar expectation $r_{\mathrm{c}} \propto M_{500}^{1 / 3}$. The solid line represents the BCES bisector fit for all 106 clusters with the form $\log _{10}\left(\frac{r_{c}}{100 \mathrm{kpc}}\right)=$ $A+B \cdot \log _{10}\left(\frac{M_{500}}{5 \times 10^{14} M_{\odot}}\right)$. The dashed and the dotted lines represent the fits for the NCCCs and CCCs, respectively (see Table 6).

radii may be larger for higher cluster masses. For the CCCs, it might be the increasing dominance of the central cluster galaxy with decreasing cluster mass that makes the core region relatively more compact for less massive systems.

It is interesting to note that the relation fitted to the complete sample is steeper than each of the separately fitted relations. This is exactly the effect mentioned above. It is the result of an offset in the relation of the two subsamples (significantly smaller core radii for the CCCs) and, in addition, of a biased distribution of the clusters in the two subsamples with more CCCs at the lowmass end and more NCCCs at the high-mass end. Among all the plots we show in this paper, this is the relation where this effect is most pronounced.

\subsection{The $M-T_{h}$ relation}

Figure 5 shows the relation of the globally measured X-ray temperature of the hot component, $T_{\mathrm{h}}$ (excluding non-ASCA derived $T_{\mathrm{h}}$ ), and the cluster mass, $M_{500}$. The self-similar model prediction is a slope with a value of 1.5 , consistent with the value we obtain for the total sample with measured $T_{\mathrm{h}}$ and consistent with the discussion in Finoguenov et al. (2001). The results of power-law fits to all the relations discussed in this section are summarized in Table 7. Moreover, the slopes and the normalizations of the $M-T_{\mathrm{h}}$ relation for the CCCs and NCCCs are consistent within errors. Thus there is no significant influence of 


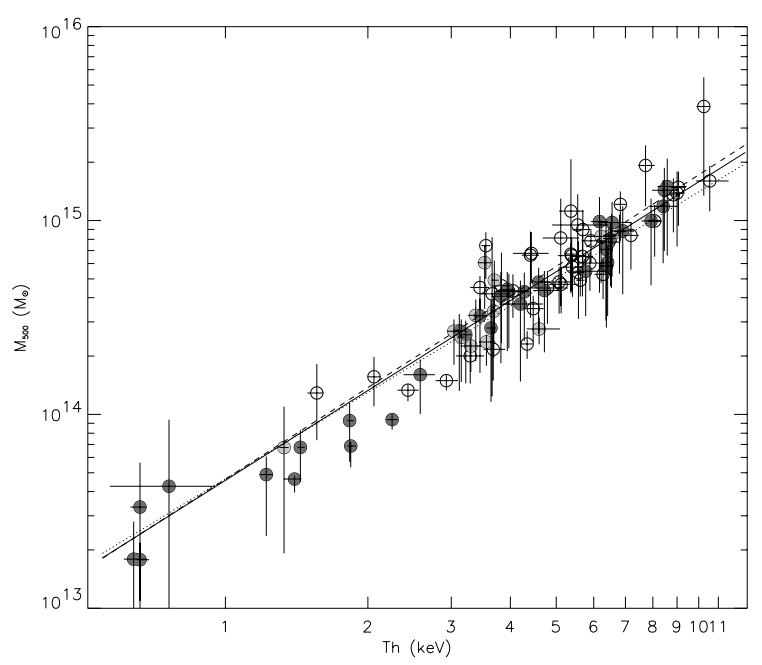

Fig. 5. The $M_{500}-T_{\mathrm{h}}$ relation. The slopes derived from the total 88 clusters with measured $T_{\mathrm{h}}$ from ASCA (solid line), CCCs (dotted line), and NCCCs (dashed line) are consistent with each other.

cooling cores on this relation. Note, however, that the two cluster parameters compared in this relation, $T_{\mathrm{h}}$ and $M_{500}$, are not independently obtained, but $M_{500}$ is directly dependent on the temperature measurements. If $T_{\mathrm{h}}$ has an offset $\Delta T_{\mathrm{h}}, M_{500}$ will change to $M_{500}+\Delta M_{500} \propto\left(T_{\mathrm{h}}+\Delta T_{\mathrm{h}}\right)^{3 / 2}$. Note that this slope is the same as the self-similar model prediction. Thus the cooling cores' influence on temperature determination will appear in the cluster mass such that the overall effect may remain undetected (see also discussion below).

Among the relations listed in Table 7 , in conjunction with the $M_{500}-T_{\mathrm{h}}$ relation, together with the $M_{\mathrm{gas}, 500}-T_{\mathrm{h}}$ relation (Fig. 11), has the smallest scatter. This could be due in part to the correlation of the mass and temperature parameter. We use the $M_{\mathrm{gas}, 500}-T_{\mathrm{h}}$ relation to show below (Sect. 4.5) that this is not a strong effect and thus not the main reason for the different $L_{X}$ normalization. Instead, the tight relation shows that mass and temperature are linked in a more fundamental way by simple self-similar gravitational processes than by the other relations.

\subsection{The $L_{X}-T$ relation}

The $L_{\mathrm{X}}-T_{\mathrm{m}}$ and $L_{\mathrm{X}}-T_{\mathrm{h}}$ relations for the cluster sample with measured $T_{\mathrm{m}}$ and $T_{\mathrm{h}}$ derived from ASCA are shown in Figs. 6 and 7. The values of $L_{\mathrm{X}}$ are the X-ray luminosities in (0.1-2.4 keV) derived from Ikebe et al. (2002). Since for some clusters $T_{\mathrm{m}}$ is measured with the central region excluded (cooling flow correction), here we only use $T_{\mathrm{m}}$ without any cooling flow correction. For $T_{\mathrm{m}}$ derived from Fukazawa et al. (1998), which includes cooling flow correction, we use the central $T_{\mathrm{m}}$ $\left(0-2^{\prime} \sim 3^{\prime}\right)$ instead (Fukazawa et al. 2000). The resulting slopes of $L_{\mathrm{X}}-T$ relations are $2.23 \pm 0.15$ and $2.73 \pm 0.13$ for $T_{\mathrm{m}}$ and $T_{\mathrm{h}}$, respectively. They are much higher than the 1.5 predicted by a self-similar model for the $L_{X}-T$ relation (note $L_{X}$ is in the ROSAT band, not bolometric). This is consistent with the results in Reiprich \& Böhringer (2002). Note that the slope of $L_{\mathrm{X}}-T_{\mathrm{m}}$ is shallower than that of $L_{\mathrm{X}}-T_{\mathrm{h}}$. This may be due to $T_{\mathrm{m}}$ having an offset to the low temperature direction compared to $T_{\mathrm{h}}$ and fewer low $L_{\mathrm{X}}$ clusters being included in the $T_{\mathrm{m}}$ relation. Remarkable is the clearly higher normalization of the relation for CCCs compared to NCCCs, with offsets of factors of 2.05 and 1.84 for the relation with $T_{\mathrm{m}}$ and $T_{\mathrm{h}}$, respectively. The normalization difference is slightly small using $T_{\mathrm{h}}$ in the scaling relation and the

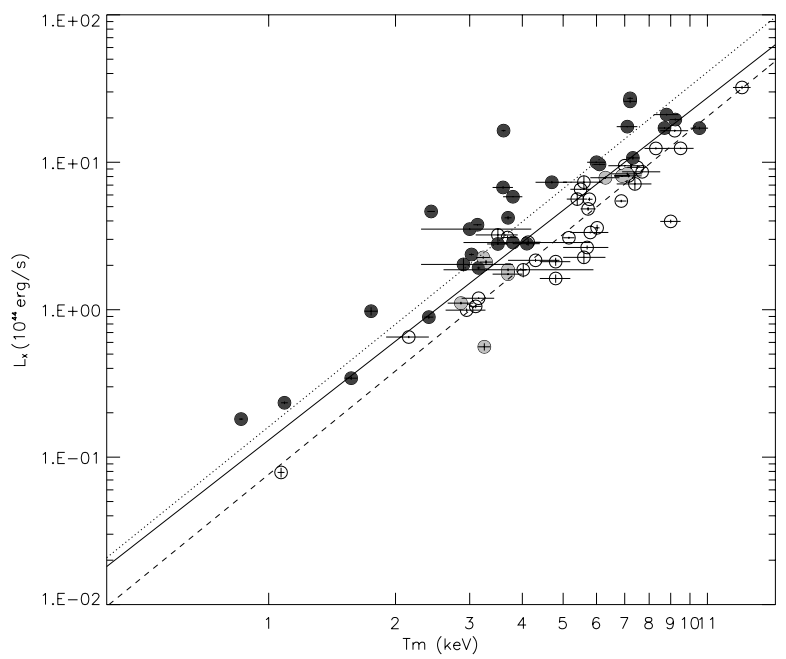

Fig. 6. The $L_{\mathrm{X}}-T_{\mathrm{m}}$ relation. The solid line shows the power-law relation fit to all the data, and the dotted and dashed lines are those for the CCCs and NCCCs, respectively. $T_{\mathrm{m}}$ with a, b, c, and d in Table 1 are selected to plot here. Note that $T_{\mathrm{m}}$ with $\mathrm{d}$ is replaced by the temperature measured from the central $2^{\prime}$ or $3^{\prime}$ region (see Table 2 in Fukazawa et al. 2000).

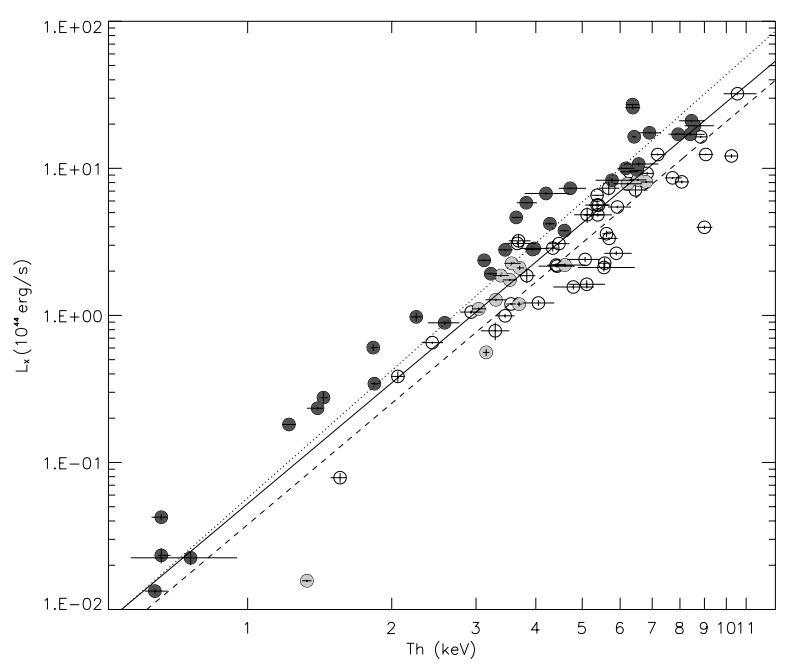

Fig. 7. The $L_{\mathrm{X}}-T_{\mathrm{h}}$ relation. The solid line shows the power-law relation fit to all the data, and the dotted and dashed lines are those for the CCCs and NCCCs, respectively.

scatter is also slightly reduced, since $T_{\mathrm{h}}$ most probably provides a better measure of the global gravitational potential depth and better mass proxy, as this temperature is not as downward-biased by the central cooling core region as $T_{\mathrm{m}}$. But the difference between the two relations is not very large. This is already an indication that biased temperature measurements for CCCs are not the major reason for the different normalizations of the relations for CCCs and NCCCs. Here the effect of the different mass coverage of CCCs and NCCCs on the slope of the combined relation (which should make the relation shallower) is not as strong as in Fig. 4. The combined relation is slightly shallower than the NCCC and CCC relations.

\subsection{The $L_{x}-M$ relation}

The $L_{X}-M$ relation is the most important relation for the application to cosmological cluster surveys. The previously determined relation for this sample (Reiprich \& Böhringer 2002) was 


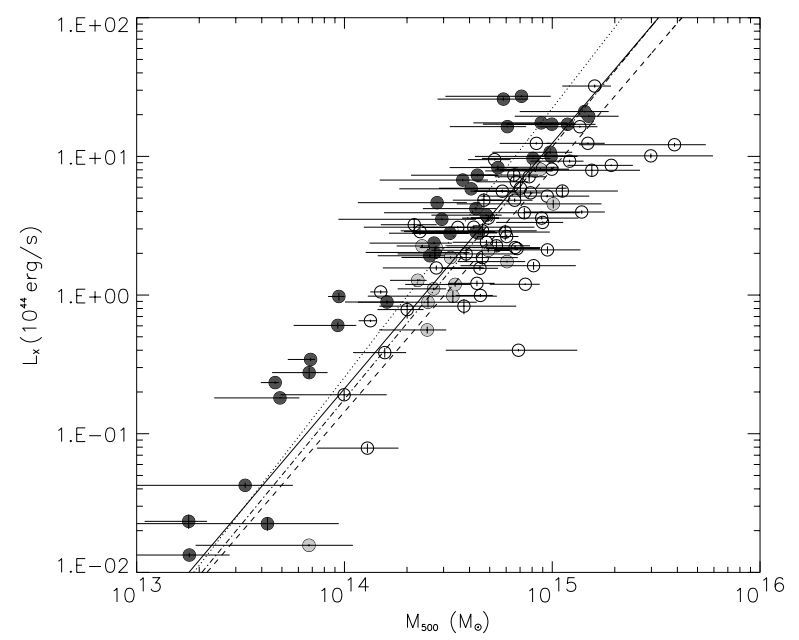

Fig. 8. The $L_{X}-M_{500}$ relation. The dot-dashed line shows the power-law relation fit to all the data (106 clusters). The solid line shows that for all the data with ASCA measured $T_{\mathrm{h}}$, and the dotted and dashed lines are those for the CCCs and NCCCs, respectively.

used to get cluster mass estimates, for example, for the cosmological studies (Schuecker et al. 2001b, 2003; Stanek et al. 2006). The resulting slope of $L_{X}-M$ relation is again higher than 1.0 , which is predicted by a self-similar model, as shown in Fig. 8.

Like in the X-ray luminosity temperature relation, we see a substantial difference in the normalization of this relation for the CCCs and NCCCs by about a factor of 2.4. The offset between the different cluster subsamples shows that the scatter in the overall sample is partly produced by the different types of clusters, and knowing more about the clusters helps reduce this scatter as discussed below.

One important question concerns the origin of this large difference. There are, in principle, two effects caused by cool cores that add to the observed result: cluster temperatures and the cluster masses derived from temperature estimates will be biased low and luminosities will be biased high due to the enhanced emission of dense cores and more compact clusters. In turn, if the core radii of the NCCCs are inflated, the cluster masses will be biased high for the NCCCs.

To distinguish these different possibilities, we need mass estimators independent of the temperature and independent of the core radii. In the next section we apply these new parameters. Figure 8 also shows that the fraction of CCCs at the high $L_{X}$ end do not constitute a small fraction of all clusters unlike that at the high $M_{500}$ end. This is due to CCCs usually having higher $L_{\mathrm{X}}$ for the clusters with the same $M_{500}$. The CCC fraction is about $60 \%$ (11 CCCs) for the most luminous 18 clusters (with $L_{\mathrm{X}}>6 \times 10^{44} \mathrm{erg} / \mathrm{s}$ and $z<0.17$ in the $\Lambda$ CDM cosmology) in this sample. This fraction is consistent with the distant cluster samples with similar $L_{\mathrm{X}}$, e.g., $7 \mathrm{CCCs}$ out of 12 clusters at $z \sim 0.2$ (Zhang et al. 2006b) and 6 CCCs out of 13 clusters at $z \sim 0.3$ (Zhang et al. 2006a), where we use the same criterion of CCCs as in the HIFLUCS. This result shows that the fraction of CCCs in luminous cluster samples does not show a large evolutionary effect up to $z \sim 0.3$.

\subsection{Relations involving total NIR luminosity and gas mass}

Assuming that the cluster gas mass fraction is approximately constant with cluster mass (e.g. Allen et al. 2004;

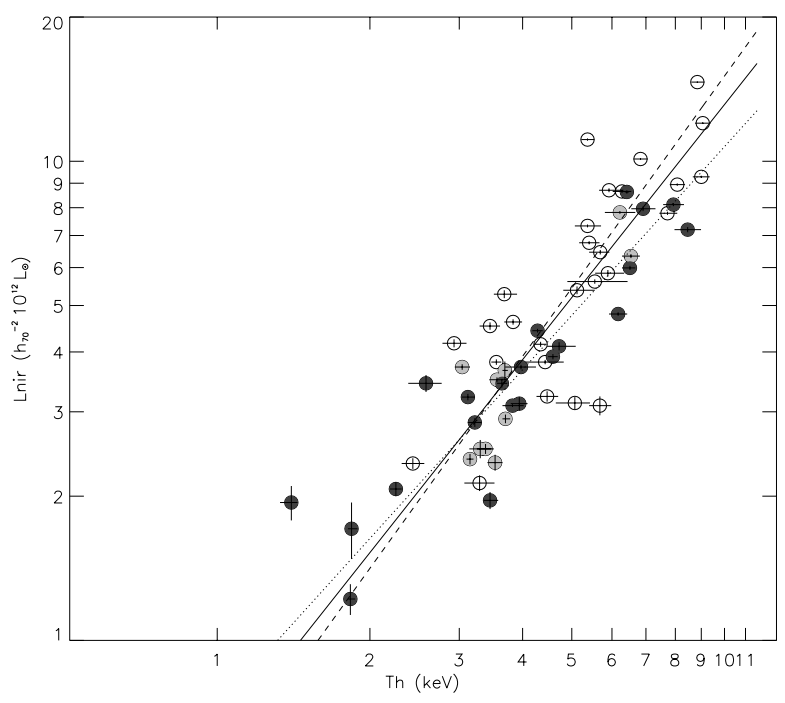

Fig. 9. The $L_{\mathrm{nir}}-T_{\mathrm{h}}$ relation. The solid line shows the power-law relation fit to all the data and the dotted and dashed lines are those for the CCCs and NCCCs, respectively.

Ettori et al. 2003), we can also use the cluster gas mass to estimate the cluster's total mass. The determination of the cluster gas mass depends only on the X-ray surface-brightness distribution, not on the temperature. However, the way we determine $M_{\mathrm{gas}, 500}$, based on the fiducial outer radius of $r_{500}$, introduces a weak temperature dependence, since in our approach we used the temperature-dependent gravitational mass to estimate $r_{500}$. This dependence is roughly proportional to $T^{1 / 2}$ and thus much weaker than proportional and much weaker than the dependence of $M_{500} \propto T^{3 / 2}$. Thus if there is any strong bias in the temperature determination due to cooling flows, we should still see this effect in a correlation analysis based on gas mass, but it will just be weakened approximately by a factor of two. For example, if the temperature of a cluster, $T$, is biased to $2 T$, the measured $M_{\text {gas,500 }}$ will change to $1.4 M_{\text {gas,500. However, if this cluster re- }}$ mains on the line with a slope 2.0 (see Table 7), it needs $M_{\text {gas,500 }}$ to change to $4 M_{\mathrm{gas}, 500}$. From this example, one can see that $M_{\mathrm{gas}, 500}$ is insensitive to $T$ in the relation $M_{\mathrm{gas}, 500}-T$. Another mass estimator is the total luminosity of the cluster galaxies in the NIR ( $K$-band), which is obtained from the Two Micron All Sky Survey (2MASS) (Lin et al. 2004).

The $L_{\mathrm{nir}}-T_{\mathrm{h}}$ relation in Fig. 9 shows no strong bias of CCCs versus NCCCs. The normalization difference is less than $10 \%$ so well within the $1 \sigma$ error of the fits. The comparison of the two mass estimators $L_{\text {nir }}$ and $M_{\text {gas,500 }}$ in Fig. 10 shows a comparatively small difference between the CCCs and NCCCs with a factor smaller than about 1.25, where the CCCs have on average a little higher $M_{\text {gas,500. }}$. Therefore we do not see a strong bias in either of the two mass estimators, although a weak bias cannot be ruled out, especially in the gas mass.

Similarly, in Figs. 11 and 12 where we show the $M_{\mathrm{gas}, 500}-T_{\mathrm{h}}$ and $M_{\mathrm{gas}, 500}-T_{\mathrm{m}}$ relations, we do not see a strong temperature bias for CCCs versus NCCCs. Similar to Figs. 6 and 7, the slope of $M_{\mathrm{gas}, 500}-T_{\mathrm{m}}$ is shallower than that of $M_{\mathrm{gas}, 500}-T_{\mathrm{h}}$, but it only has a slight difference for the clusters with $T_{\mathrm{h}}>3 \mathrm{keV}$. Note that clusters with $T_{\mathrm{m}}<1.0 \mathrm{keV}$ in Fig. 11 are not plotted in Fig. 12. In addition, the difference in the normalization of this relation for the CCCs and NCCCs is very small and within the measurement errors, with a factor 1.10 and 1.00 for $T_{\mathrm{m}}$ and $T_{\mathrm{h}}$, respectively. Therefore we conclude from the results in this section that the segregation of CCCs and NCCCs in the $L_{X}-M$ and 


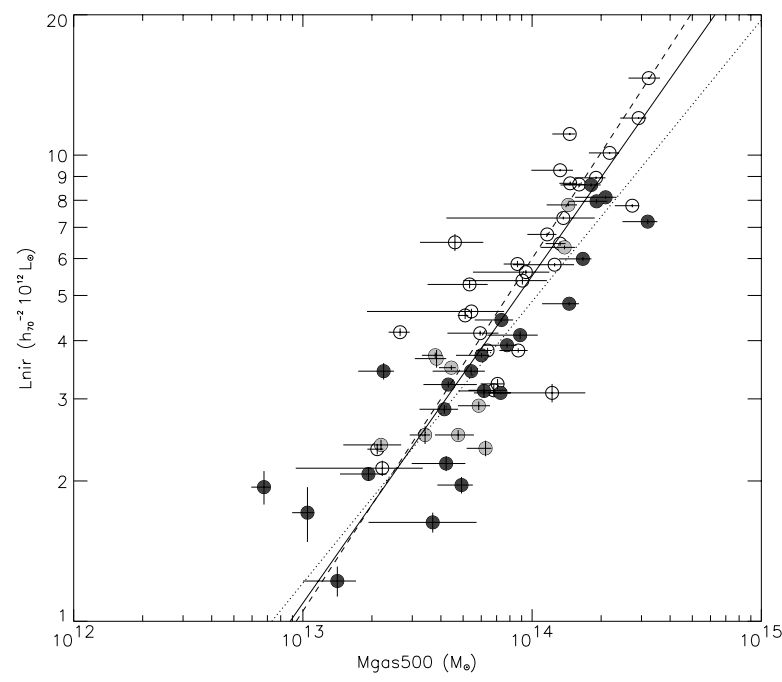

Fig. 10. The $L_{\text {nir }}-M_{\text {gas } 500}$ relation. The solid line shows the power-law relation fit to all the data, and the dotted and dashed lines are those for the CCCs and NCCCs, respectively.

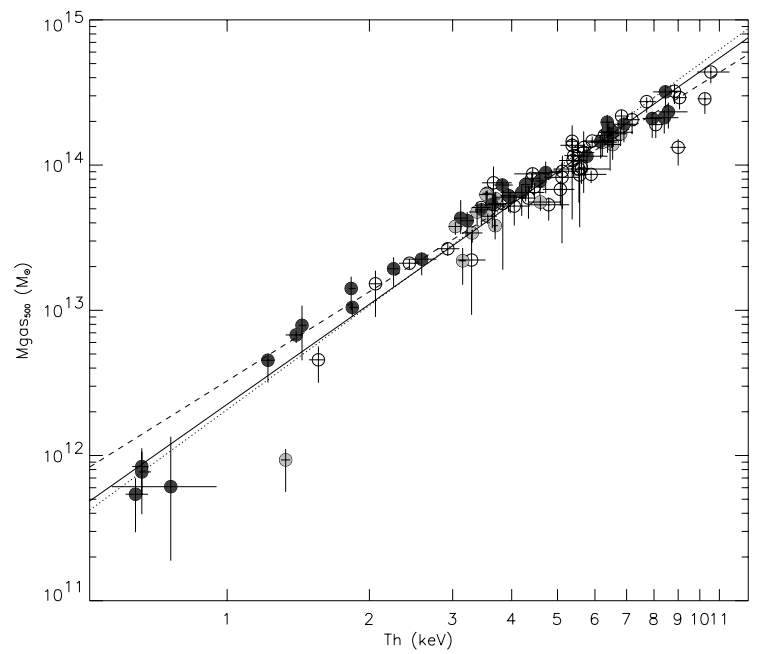

Fig. 11. The $M_{\mathrm{gas}, 500}-T_{\mathrm{h}}$ relation. The solid line shows the power-law relation fit to all the data, and the dotted and dashed lines are those for the CCCs and NCCCs, respectively.

$L_{\mathrm{X}}-T$ relations is mainly an X-ray luminosity effect and, to a lesser extent, an effect of a biased temperature estimate. A similar conclusion has been reached by O'Hara et al. (2006).

\subsection{Gas mass fraction}

We found no difference of the total gas mass fraction, $f_{\text {gas, } 500}$, between CCCs and NCCCs as shown in Fig. 13. This reconfirms the weak influence of cooling cores on the mass and gas mass estimates.

\section{Discussions and summary}

In this paper we have used an isothermal model to determine the X-ray mass, because to date only global temperature estimates mainly from ASCA are available for such a large sample. For the central region in cooling core clusters, this is obviously not correct. But we expect that the total gravitational mass is correct at large radii, which is confirmed by our mass determination for the cluster PKS 0745-191 and Abell 1650 based on

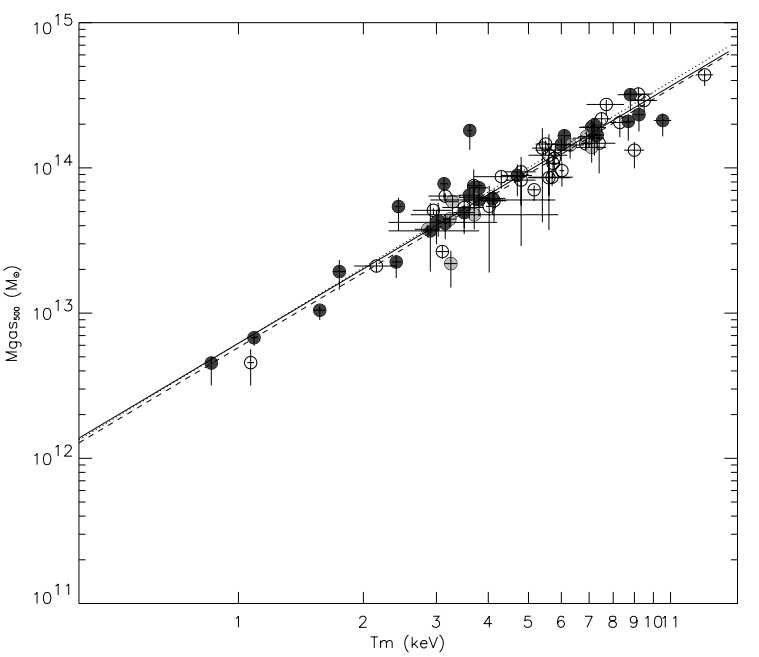

Fig. 12. The $M_{\text {gas,500 }}-T_{\mathrm{m}}$ relation. $T_{\mathrm{m}}$ with a, b, c and d in Table 1 are selected to plot here. Note $T_{\mathrm{m}}$ with $\mathrm{d}$ is replaced by the temperature measured from the central $2^{\prime}$ or $3^{\prime}$ region (see Table 2 in Fukazawa et al. 2000). Symbols have the same meanings as in Fig. 11.

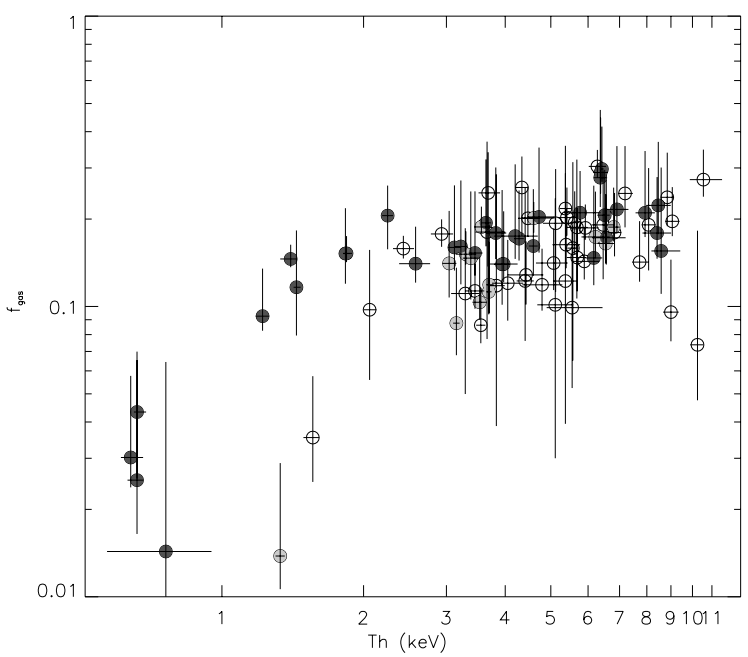

Fig. 13. The $f_{\text {gas }, 500}-T_{\mathrm{h}}$ relation.

XMM-Newton observations (Chen et al. 2003; Jia et al. 2006) in which the resultant total masses are found to be consistent with this work. that:

In summary we find from the analysis presented in this paper

(i) The formally-derived mass deposition rates for the strongest cooling core clusters are roughly proportional to the cluster mass.

(ii) The fraction of NCCCs increases significantly with $M_{500}$, and most of the galaxy groups in HIFLUGCS are cooling core clusters. This is most probably explained by the fact that the most massive galaxy clusters have been formed more recently than the others and should therefore show a larger fraction of dynamically young systems. These may turn into cooling core clusters in a later evolutionary stage. In addition, the fraction of CCCs in luminous cluster samples does not show a large evolution effect within $z<0.3$.

(iii) Among all the observational parameters, the core radius and the X-ray luminosity are shown to be most affected by the presence of a cooling core, as observed in their 
relation to other bulk cluster properties as cluster mass and temperature.

(iv) The $M-T$ relation using the X-ray temperature of the hot ICM phase, $T_{\mathrm{h}}$, seems to show a comparatively small bias for CCCs in comparison to NCCCs.

From the magnitude of the effect (iii) we can conclude that the scatter in the $L_{X}-M_{500}$ relation, which is so important in cosmological applications, is to a large part due to the different normalizations of CCCs and NCCCs. It is important to distinguish between statistical, systematic, and intrinsic scatter and to take the intrinsic scatter into account in applications (e.g. Ikebe et al. 2002; Stanek et al. 2006). The findings here indicate that a significant portion of the scatter may be intrinsic due to variations in the X-ray luminosity for clusters of a given mass. We are currently working to confirm this result with high quality Chandra and XMM-Newton observations of the complete HIFLUGCS sample. If a substantial scatter is confirmed, it will be interesting to check if these results are fully consistent with the low $\Omega_{\mathrm{m}}$ and $\sigma_{8}$ values found from the WMAP 3rd year data (Spergel et al. 2006) combined with cluster mass-function prediction and observed luminosity function (Reiprich 2006). This finding also points the way to an improvement in the $L_{X}-M_{500}$ relation. Given a good proxy for the strength of cooling cores, one could correct for this effect and tighten this important relation. This was indeed suggested by O'Hara et al. (2006) who propose to use the central surface brightness as such a proxy. Further work is in progress to use the HIFLUGCS cluster sample to work out a correction scheme.

Acknowledgements. Y. Chen was supported by the exchange program between the Max-Planck Society and the Chinese Academy of Sciences. He thanks MPE for its very gracious hospitality. This research is partially supported by the Scientific Research Foundation for the Returned Overseas Chinese Scholars, State Education Ministry. T.H.R. acknowledges support by the Deutsche Forschungsgemeinschaft through Emmy Noether Research grant RE 1462.

\section{References}

Akritas, M. G., \& Bershady, M. A. 1996, ApJ, 470, 706

Allen, S. W., Schmidt, R. W., Ebeling, H., Fabian, A. C., \& van Speybroeck, L. 2004, MNRAS, 353, 457

Arnaud, K. A. 1996, ADASS, 101, 5

Arnaud, M., Pointecouteau, E., \& Pratt, G. W. 2005, A\&A, 441, 893

Böhringer, H., Matsushita, K., Churazov, E., Ikebe, Y., \& Chen, Y. 2002, A\&A, 382,804
Cavaliere, A., \& Fusco-Femiano, R. 1976, A\&A, 49, 137

Chen, Y., Ikebe, Y., \& Böhringer, H. 2003, A\&A, 407, 41

David, L. P., Nulsen, P. E. J., \& McNamara, B. R. 2001, ApJ, 557, 546

Edge, A. C., \& Stewart, G. C. 1991, MNRAS, 252, 414

Ettori, S. 2000, MNRAS, 311, 313

Ettori, S., De Grandi, S., \& Molendi, S. 2002, A\&A, 391, 841

Ettori, S., Tozzi, P., \& Rosati, P. 2003, A\&A, 398, 879

Fabian, A. C. 1994, ARA\&A, 32, 277

Fabian, A. C., Crawford, C. S., Edge, A. C., \& Mushotzky, R. F. 1994, MNRAS, 267,779

Fabian, A. C., Sanders, J. S., Allen, S. W., et al. 2003, MNRAS, 344, L43

Finoguenov, A., Reiprich, T. H., \& Böhringer, H. 2001, A\&A, 368, 749

Fukazawa, Y., Makishima, K., Tamura, T., et al. 1998, PASJ, 50, 187

Fukazawa, Y., Makishima, K., Tamura, T., et al. 2000, MNRAS, 313, 21

Ikebe, Y., Makishima, K., Fukazawa, Y., et al. 1999, ApJ, 525, 58

Ikebe, Y., Reiprich, T. H., Böhringer, H., et al. 2002, A\&A, 383, 773

Jia, S. M., Chen, Y., \& Chen, L. 2006, ChJAA, 6, 181

Jones, C., \& Forman, W. 1984, ApJ, 276, 38

Lin, Y. T., Mohr, J. J., \& Stanford, S. A. 2004, ApJ, 617, 879

Makishima, K., Ezawa, H., Fukuzawa, Y., et al. 2001, PASJ, 53, 401

Markevitch, M. 1998, ApJ, 504, 27

Markevitch, M., Forman, W. R., Sarazin, C. L., \& Vikhlinin, A. 1998, ApJ, 503, 77

McCarthy, I. G., Balogh, M. L., Babul, A., Poole, G. B., \& Horner, D. J. 2004, ApJ, 613, 811

McNamara, B. R., Nulsen, P. E. J., Wise, M. W., et al. 2005, Nature, 433, 45

Molendi, S., \& Pizzolato, F. 2001, ApJ, 560, 194

O’Hara, T. B., Mohr, J. J., Bialek, J. J., \& Evrard, A. E. 2006, ApJ, 639, 640

Ota, N., \& Mitsuda, K. 2004, A\&A, 428, 757

Peres, C. B., Fabian, A. C., Edge, A. C., et al. 1998, MNRAS, 298, 416

Peterson, J. R., Paerels, F. B. S., Kaastra, J. S., et al. 2001, A\&A, 365, L104

Peterson, J. R., Kahn, S. M., Paerels, F. B. S., et al. 2003, ApJ, 590, 207

Pratt, G. W., et al. 2006, A\&A, submitted

Reiprich, T. H. 2001, Cosmological Implications and Physical Properties of an X-ray Flux-Limited Sample of Galaxy Clusters, Ph.D. Thesis

Reiprich, T. H. 2006, A\&A, 453, L39

Reiprich, T. H., \& Böhringer, H. 2002, ApJ, 567, 716

Schuecker, P., Böhringer, H., Reiprich, T. H., \& Feretti, L. 2001a, A\&A, 378, 408

Schuecker, P., Böhringer, H., Guzzo, L., et al. 2001b, A\&A, 368, 86

Schuecker, P., Böhringer, H., Collins, C. A., \& Guzzo, L. 2003, A\&A, 398, 867

Spergel, D. N., Beam, R., Doré, O., et al. 2006, ApJ, submitted [arXiv: astro-ph/0603449]

Stanek, R., Evrard, A. E., Böhringer, H., Schuecker, P., \& Nord, B. 2006, ApJ, 648,956

Vikhlinin, A., Markevitch, M., Murray, S. S., et al. 2005, ApJ, 628, 655

Voit, M. 2005, Rev. Mod. Phys., 77, 207

White, D. A. 2000, MNRAS, 312, 663

White, D. A., Jones, C., \& Forman, W. 1997, MNRAS, 292, 419

Zhang, Y. Y., Böhringer, H., Finoguenov, A., et al. 2006a, A\&A, 456, 55

Zhang, Y. Y., Finoguenov, A., Böhringer, H., et al. 2006b, preprint 
Y. Chen et al.: Statistics of X-ray observables of galaxy clusters, Online Material $p 1$

\section{Online Material}


Y. Chen et al.: Statistics of X-ray observables of galaxy clusters, Online Material $p 2$

Table 1. Cluster properties.

\begin{tabular}{|c|c|c|c|c|c|c|c|}
\hline Name & $z$ & $T_{\mathrm{m}}(\mathrm{keV})$ & $\beta$ & $r_{\mathrm{c}}(\mathrm{kpc})$ & $n_{\text {center }}\left(10^{-2} \mathrm{~cm}^{-3}\right)$ & $t_{\text {cool }}\left(10^{10} \mathrm{yr}\right)$ & $\dot{M}\left(M_{\odot} / \mathrm{yr}\right)$ \\
\hline $2 \mathrm{~A} 0335$ & 0.0349 & $d 3.01_{-0.07}^{+0.07}$ & $0.575_{-0.003}^{+0.004}$ & $33_{-0}^{+0}$ & $5.47_{-0.06}^{+0.06}$ & $0.10_{-0.00}^{+0.00}$ & $360_{-17}^{+20}$ \\
\hline A0085 & 0.0556 & ${ }^{a} 6.10_{-0.20}^{+0.20}$ & $0.532_{-0.004}^{+0.003}$ & $82_{-3}^{+3}$ & $2.57_{-0.10}^{+0.010}$ & $0.33_{-0.02}^{+0.00}$ & $200_{-27}^{+33}$ \\
\hline A0119 & 0.0440 & ${ }^{a} 5.80_{-0.60}^{+0.60}$ & $0.675_{-0.023}^{+0.004}$ & $501_{-26}^{+27}$ & $0.15_{-0.01}^{+0.01}$ & $5.49_{-0.058}^{+0.62}$ & $0_{-0}^{+0}$ \\
\hline A0133 & 0.0569 & $c 3.80_{-0.90}^{+2.00}$ & $0.530_{-0.004}^{+0.023}$ & $45_{-1}^{+1}$ & $2.80_{-0.08}^{+0.08}$ & $0.23_{-0.04}^{+0.09}$ & $108_{-51}^{-0.55}$ \\
\hline A0262 & 0.0161 & $d_{2} .15_{-0.06}^{+0.00}$ & $0.443_{-0.017}^{+0.018}$ & $41_{-9}^{+11}$ & $0.81_{-0.09}^{+0.13}$ & $0.56_{-0.08}^{+0.04}$ & $14_{-4}^{+41}$ \\
\hline A0399 & 0.0715 & $a_{7.40_{-0.70}^{+0.90}}$ & $0.713_{-0.095}^{+0.137}$ & $449_{-99}^{+131}$ & $0.22_{-0.03}^{+0.89}$ & $4.32_{-0.070}^{+0.88}$ & $0_{-0}^{+0^{4}}$ \\
\hline A0400 & 0.0240 & ${ }^{d} 2.31_{-0.14}^{+0.14}$ & $0.534_{-0.013}^{+0.8914}$ & $154_{-8}^{+9}$ & $0.20_{-0.01}^{+0.01}$ & $2.39_{-0.12}^{+0.13}$ & $0_{-0}^{+0}$ \\
\hline A0401 & 0.0748 & ${ }^{a} 8.30_{-0.50}^{+0.50}$ & $0.613_{-0.010}^{+0.013}$ & $245_{-10}^{+8}$ & $0.60_{-0.04}^{+0.05}$ & $1.67^{-0.15}$ & $0_{-0}^{+0}$ \\
\hline A0478 & 0.0900 & $a^{a} .10_{-0.40}^{+0.40}$ & $0.613_{-0.004}^{+0.8004}$ & $98_{-2}^{+2}$ & $3.55_{-0.014}^{+0.04}$ & $0.26_{-0.02}^{+0.02}$ & $645_{-113}^{+137}$ \\
\hline A0496 & 0.0328 & ${ }^{d} 4.13_{-0.08}^{+0.08}$ & $0.484_{-0.003}^{+0.004}$ & $30_{-1}^{+1}$ & $4.07_{-0.26}^{+0.14}$ & $0.17_{-0.01}^{+0.01}$ & $114_{-28}^{-113}$ \\
\hline A0576 & 0.0381 & ${ }^{b} 4.02_{-0.07}^{+0.08}$ & $0.825_{-0.185}^{+0.432}$ & $394_{-125}^{+221}$ & $0.16_{-0.04}^{+0.06}$ & $4.27_{-1.09}^{+1.01}$ & $0_{-0}^{+-28}$ \\
\hline A0754 & 0.0528 & ${ }^{a} 9.00_{-0.50}^{+0.50}$ & $0.698_{-0.024}^{+0.027}$ & $239_{-16}^{+175}$ & $0.44_{-0.02}^{+0.04}$ & $2.41_{-0.13}^{+0.13}$ & $0_{-0}^{+0}$ \\
\hline A1060 & 0.0114 & $d 3.24_{-0.06}^{+0.00}$ & $0.607_{-0.034}^{-0.024}$ & $94_{-12}^{+15}$ & $0.47_{-0.04}^{+0.02}$ & $1.29_{-0.11}^{+0.13}$ & $\begin{array}{l}-0 \\
0_{-0}^{+0}\end{array}$ \\
\hline A1367 & 0.0216 & $d 3.55_{-0.08}^{+0.00}$ & $0.695_{-0.032}^{+0.034}$ & $383_{-22}^{+24}$ & $0.15_{-0.01}^{-0.04}$ & $4.29_{-0.21}^{+0.21}$ & $0_{-0}^{+0}$ \\
\hline A1644 & 0.0474 & $4.70_{-0.70}^{+0.90}$ & $0.579_{-0.074}^{+0.0111}$ & $299_{-92}^{+122}$ & $0.28_{-0.06}^{+0.01}$ & $2.65_{-0.07}^{+0.91}$ & $0_{-0}^{+0}$ \\
\hline A 1650 & 0.0845 & $\begin{array}{r}-0.70 \\
{ }^{a} 5.60_{-0.60}^{+0.60}\end{array}$ & $0.704_{-0.081}^{+0.074}$ & $281_{-70}^{+102}$ & $0.43_{-0.06}^{+0.06}$ & $1.87_{-0.36}^{+0.67}$ & $0_{-0}^{+0}$ \\
\hline A1651 & 0.0860 & ${ }^{a} 6.30_{-0.050}^{+0.50}$ & $0.643_{-0.013}^{+0.0814}$ & $\begin{array}{r}-70 \\
180_{-9}^{+9}\end{array}$ & $\begin{array}{l}-0.06 \\
1.10_{-0.07}^{+0.07}\end{array}$ & $0.78_{-0.07}^{+0.076}$ & $81_{-23}^{+03}$ \\
\hline A 1736 & 0.0461 & $a_{3.50_{-0.0}^{+0.40}}$ & $0.542_{-0.092}^{+0.0137}$ & $374_{-129}^{+177}$ & $0.13_{-0.00}^{+0.07}$ & $4.92_{-128}^{-0.07}$ & $0_{-0}^{+03}$ \\
\hline A1795 & 0.0616 & ${ }^{a} 6.00_{-0.030}^{+0.30}$ & $0.596_{-0.002}^{+0.092}$ & $78_{-1}^{+129}$ & $2.88_{-0.05}^{+0.02}$ & $0.29_{-0.01}^{+0.01}$ & $399_{-39}^{+4}$ \\
\hline A2029 & 0.0767 & $\begin{array}{r}0.00-0.30 \\
a \\
8.70_{-0.30}^{+0.30}\end{array}$ & $0.582_{-0.004}^{+0.002}$ & $83_{-2}^{+2}$ & $3.90_{-0.015}^{+0.05}$ & $0.26_{-0.01}^{+0.01}$ & $514_{-78}^{+393}$ \\
\hline A2052 & 0.0348 & $\begin{array}{r}b_{3} 3.03_{-0.04}^{+0.040} \\
\end{array}$ & $0.526_{-0.005}^{+0.004}$ & $36_{-1}^{+1}$ & $2.85_{-0.02}^{+0.66}$ & $\begin{array}{l}0.00_{-0.01}^{+0.06} \\
0.20_{-0.04}^{+0.0}\end{array}$ & $108_{-49}^{+188}$ \\
\hline A2063 & 0.0354 & 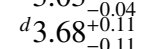 & $0.561_{-0.011}^{+0.005}$ & $\begin{array}{c}10_{-1}^{+1} \\
110_{-6}^{+6}\end{array}$ & $0.96_{-0.00}^{+0.62}$ & $\begin{array}{l}0.67_{-0.02}^{+0.04} \\
0.02\end{array}$ & $21_{-2}^{+2}$ \\
\hline A2065 & 0.0721 & $\begin{array}{r}r .00_{-0.11} \\
a 5.40_{-0.30}^{+0.30}\end{array}$ & $\begin{array}{l}0.162_{-0282}^{+0.011} \\
1.734\end{array}$ & $689_{-186}^{+360}$ & $\begin{array}{l}0.0 .00 \\
0.20_{-0.04}^{+0.07}\end{array}$ & $\begin{array}{l}0.01-0.02 \\
3.99_{-1.154}^{+1.15}\end{array}$ & $\begin{array}{c}c_{-2} \\
0_{-0}^{+0}\end{array}$ \\
\hline A 2142 & 0.0899 & $\begin{array}{l}-0.30 \\
a 8.80_{-0.60}^{+0.60}\end{array}$ & $0.591_{-0.006}^{+0.082}$ & $153_{-5}^{+56}$ & $\begin{array}{l}0.0 .04 \\
1.61^{+0.07}\end{array}$ & $0.64^{+0.04}$ & $337^{-0}+82$ \\
\hline A 2147 & 0.0351 & $\begin{array}{r}0.00_{-0.60} \\
d_{4} .91_{-028}^{+0.28}\end{array}$ & $0.444_{-0.041}^{+0.006}$ & $237_{-64}^{+102}$ & $\begin{array}{l}1.01-0.07 \\
0.17_{-0.03}^{+0.04}\end{array}$ & $\begin{array}{l}0.41_{-0.87}^{+0.94} \\
4.84\end{array}$ & $0_{-0}^{+01}$ \\
\hline A2163 & 0.2010 & ${ }^{b} 13.29_{-0.64}^{+0.28}$ & $0.796_{-0.028}^{+0.046}$ & $519_{-29}^{+64}$ & $0.53_{-0.02}^{+0.03}$ & $2.37_{-0.11}^{+0.87}$ & $\begin{array}{l}-0 \\
0_{-0}^{+0}\end{array}$ \\
\hline A2199 & 0.0302 & $d_{4} 10_{-0.08}^{+0.084}$ & $0.655_{-0.020}^{+0.028}$ & $139_{-9}^{+10}$ & $0.83^{+0.02}$ & $\begin{array}{l}2.53^{-0.11} \\
0.03\end{array}$ & $\begin{array}{l}-0 \\
77^{+7}\end{array}$ \\
\hline A2204 & 0.1523 & ${ }^{b} 7.21_{-0.025}^{+0.08}$ & $0.597_{-0.007}^{+0.021}$ & $\begin{array}{r}-9 \\
67_{-2}^{+3}\end{array}$ & $5.07_{-0.011}^{+0.03}$ & $0.18_{-0.01}^{+0.03}$ & $1287_{-129}^{-6}$ \\
\hline A2244 & 0.0970 & $7.10_{-2,20}^{+5.250}$ & $0.607_{-0.015}^{+0.007}$ & $125_{-10}^{+10}$ & $\begin{array}{r}-0.11 \\
1.20_{-0.05}^{+0.06}\end{array}$ & $\begin{array}{l}0.10_{-0.01}^{+0.35} \\
0.76_{-018}^{+0.35}\end{array}$ & $\begin{array}{l}1201-129 \\
129^{+199}\end{array}$ \\
\hline A2255 & 0.0800 & $\begin{array}{r}{ }^{b} 6.87_{-0.20}^{+0.20} \\
6\end{array}$ & $\begin{array}{l}0.797_{-0.030}^{+0.015} \\
0.033\end{array}$ & $593_{-32}^{+35}$ & $0.18_{-0.02}^{+0.05}$ & $4.96_{-0.04}^{+0.18}$ & $\begin{array}{c}-127 \\
0_{-0}^{+0}\end{array}$ \\
\hline A2256 & 0.0601 & $\begin{array}{r}a \\
{ }^{2}\end{array}$ & $0.914_{-0.047}^{+0.030}$ & $587_{-36}^{+39}$ & $0.26_{-0.01}^{+0.02}$ & $3.62_{-021}^{+0.44}$ & $0_{-0}^{+0}$ \\
\hline A2589 & 0.0416 & ${ }^{c} 3.70_{-1.10}^{+2.20}$ & $0.596_{-0.012}^{+0.047}$ & $118_{-7}^{-36}$ & $0.74_{-0.07}^{+0.01}$ & $0.88_{-0.24}^{+0.31}$ & $\begin{array}{l}-0 \\
19_{-19}^{+53}\end{array}$ \\
\hline A2597 & 0.0852 & $a_{3} .60_{-0.20}^{+0.10}$ & $0.633_{-0.008}^{+0.0012}$ & $57_{-2}^{+2}$ & $3.63_{-0.07}^{+0.07}$ & $0.17_{-0.01}^{+0.04}$ & $501_{-51}^{-19}$ \\
\hline A2634 & 0.0312 & $\begin{array}{r}d \\
d .70_{-0.28}^{+0.20}\end{array}$ & $0.640_{-0.043}^{+0.008}$ & $364_{-38}^{+2}$ & $0.24_{-0.02}^{+0.07}$ & $\begin{array}{r}-0.01 \\
2.70_{-0.25}^{+0.28}\end{array}$ & $0_{-0}^{+0}$ \\
\hline A2657 & 0.0404 & ${ }^{a} 3.70_{-0.30}^{+0.38}$ & $0.556_{-0.007}^{+0.043}$ & $119_{-4}^{+5}$ & $0.54_{-0.00}^{-0.02}$ & $1.20_{-0.07}^{+0.05}$ & $2_{-2}^{+0}$ \\
\hline A 3112 & 0.0750 & ${ }^{a} 4.70_{-0.40}^{+0.40}$ & $0.576_{-0.006}^{+0.007}$ & $61_{-2}^{+2^{-4}}$ & $3.52_{-0.23}^{+0.20}$ & $0.21_{-0.02}^{+0.03}$ & $346_{-97}^{+123}$ \\
\hline A3158 & 0.0590 & $b_{5} .77_{-0.05}^{+0.10}$ & $0.661_{-0.022}^{+0.025}$ & $268_{-18}^{+19}$ & $0.39_{-0.01}^{+0.02}$ & $2.11_{-0.08}^{+0.08}$ & $0_{-0}^{+0}$ \\
\hline A 3266 & 0.0594 & $a_{7.70_{-0.80}^{+0.80}}^{+0.80}$ & $0.796_{-0.019}^{+0.022}$ & $564_{-19}^{+18}$ & $0.28_{-0.01}^{+0.01}$ & $3.44_{-0.30}^{+0.30}$ & $0_{-0}^{+0}$ \\
\hline A3376 & 0.0455 & ${ }^{a} 4.30_{-0.60}^{+0.60}$ & $1.054_{-0.083}^{+0.1019}$ & $754_{-60}^{+68}$ & $0.10_{-0.00}^{+0.01}$ & $6.87_{-0.88}^{+0.83}$ & $0_{-0}^{+0}$ \\
\hline A3391 & 0.0531 & $a_{5} .70_{-070}^{+0.70}$ & $0.579_{-0.024}^{+0.026}$ & $234_{-21}^{+23}$ & $0.43_{-0.08}^{+0.00}$ & $1.91_{-0.34}^{+0.38}$ & $0_{-0}^{+0}$ \\
\hline A3395s & 0.0498 & ${ }^{a} 4.80_{-0.40}^{+0.40}$ & $0.964_{-0.0167}^{+0.024}$ & $604_{-117}^{+172}$ & $0.13_{-0.02}^{+0.02}$ & $5.82_{-0.92}^{+1.01}$ & $0_{-0}^{+0}$ \\
\hline A 3526 & 0.0103 & $3.68_{-0.06}^{+0.06}$ & $0.495_{-0.010}^{+0.011}$ & $37^{+4}$ & $1.83_{-0.12}^{+0.02}$ & $0.36_{-0.00}^{+0.032}$ & $24_{-5}^{-0}$ \\
\hline A 3558 & 0.0480 & ${ }^{a} 5.50_{-0.030}^{+0.06}$ & $0.580_{-0.005}^{+0.010}$ & $223_{-5}^{-4}$ & $0.46_{-001}^{+0.12}$ & $1.77_{-0.09}^{-0.02}$ & $0_{-0}^{+0^{-5}}$ \\
\hline A3562 & 0.0499 & ${ }^{b} 5.16_{-0.16}^{+0.16}$ & $0.472_{-0.006}^{+0.003}$ & $98_{-5}^{+5^{3}}$ & $0.58_{-0.02}^{+0.01}$ & $1.33_{-0.06}^{+0.09}$ & $0_{-0}^{+0}$ \\
\hline A3571 & 0.0397 & ${ }^{a} 6.90_{-0.30}^{+0.30}$ & $0.613_{-0.010}^{+0.010}$ & $181_{-6}^{+5}$ & $1.09_{-0.08}^{+0.02}$ & $0.84_{-0.07}^{+0.06}$ & $35_{-10}^{+11}$ \\
\hline A3581 & 0.0214 & $1.83_{-0.04}^{+0.04}$ & $0.543_{-0.022}^{+0.024}$ & $35_{-4}^{+4^{-6}}$ & $1.61_{-0.12}^{+0.12}$ & $0.25_{-0.02}^{+0.03}$ & $49^{+12}$ \\
\hline A3667 & 0.0560 & $a^{2} 7.00_{-0.60}^{+0.04}$ & $0.541_{-0.008}^{+0.008}$ & $279_{-9}^{+4}$ & $0.33_{-0.01}^{+0.01}$ & $2.81_{-0.017}^{+0.02}$ & $0_{-0}^{+11}$ \\
\hline A4038 & 0.0283 & $b_{3} 3.15_{-0.03}^{+0.00}$ & $0.541_{-0.008}^{+0.008}$ & $58_{-3}^{+3}$ & $1.49_{-0.09}^{+0.09}$ & $0.40_{-0.02}^{+0.02}$ & $68^{-1}+14$ \\
\hline A4059 & 0.0460 & $a_{4} .10_{-0.30}^{+0.30}$ & $0.582_{-0.010}^{+0.010}$ & $89^{-3}$ & $1.18_{-0.08}^{+0.09}$ & $0.58_{-0.05}^{+0.02}$ & $69_{-15}^{+20}$ \\
\hline COMA & 0.0232 & ${ }^{d} 8.38_{-0.034}^{+0.34}$ & $0.654_{-0.021}^{+0.010}$ & $343_{-20}^{+5}$ & $0.30_{-0.06}^{+0.08}$ & $3.45_{-0.06}^{+0.85}$ & $0_{-0}^{+0}$ \\
\hline EXO0422 & 0.0390 & ${ }^{c} 2.90_{-0.00}^{+0.94}$ & $0.722_{-0.074}^{+0.02104}$ & $142_{-30}^{+40}$ & $0.66_{-0.00}^{+0.06}$ & $0.85_{-019}^{+0.23}$ & $48_{-38}^{+59}$ \\
\hline FORNAX & 0.0046 & ${ }^{d} 1.20_{-0.04}^{+0.00}$ & $0.804_{-0.084}^{+0.071}$ & $173_{-15}^{+17}$ & $0.09_{-0.01}^{+0.08}$ & $\begin{array}{l}2.50_{-0.18}^{+0.20} \\
2.19\end{array}$ & $0_{-0}^{+-38}$ \\
\hline HYDRA-A & 0.0538 & a $3.80^{+0.20}$ & $0.573_{-0.003}^{+0.084}$ & $50^{+1}$ & $3.58^{+0.01}+37$ & $0.18_{-0.02}^{+0.18}$ & $293_{-84}^{-150}$ \\
\hline IIIZw54 & 0.0311 & $\left(2.16_{-0.30}^{+0.35}\right)$ & $0.887_{-0.153}^{+0.323}$ & $289_{-72}^{+123}$ & $0.20^{+0.05}$ & $2.31^{+0.55}$ & $\begin{array}{c}0_{-8}^{+04} \\
-0\end{array}$ \\
\hline MKW3S & 0.0450 & $\begin{array}{l}a .030 \\
a .50_{-02}^{+0.20}\end{array}$ & $0.581_{-0.007}^{+0.008}$ & $66_{-2}^{+2}$ & $1.89_{-0.25}^{+0.23}$ & $0.33_{-0.04}^{+0.06}$ & $121_{-44}^{-7}$ \\
\hline MKW4 & 0.0200 & ${ }^{d} 1.71^{-0.09}+09$ & $0.440_{-0.005}^{+0.007}$ & $11^{+2}$ & $2.92^{-0.025}$ & $0.13^{-0.04}$ & $16^{+24}$ \\
\hline MKW8 & 0.0270 & $\begin{array}{r}-0.09 \\
3.29_{-022}^{+0.23}\end{array}$ & $0.511_{-0.059}^{+0.005}$ & $106_{-42}^{+70}$ & $\begin{array}{l}-0.09 \\
0.26_{-0.05}^{+0.11}\end{array}$ & $2.31_{-0.09}^{+0.01}$ & $0_{-0}^{+0}$ \\
\hline NGC 1550 & 0.0123 & $1.43_{-0.03}^{+0.02}$ & $0.554_{-0.037}^{+0.059}$ & $\begin{array}{l}45_{-10}^{-15} \\
\text { (15 }\end{array}$ & $0.75_{-0.09}^{+0.05}$ & $0.41_{-0.06}^{+0.09}$ & $20_{-8}^{+10}$ \\
\hline
\end{tabular}


Table 1. continued.

\begin{tabular}{cccccccc}
\hline \hline Name & $z$ & $T_{\mathrm{m}}(\mathrm{keV})$ & $\beta$ & $r_{\mathrm{c}}(\mathrm{kpc})$ & $n_{\text {center }}\left(10^{-2} \mathrm{~cm}^{-3}\right)$ & $t_{\text {cool }}\left(10^{10} \mathrm{yr}\right)$ & $\dot{M}\left(M_{\odot} / \mathrm{yr}\right)$ \\
\hline NGC 4636 & 0.0037 & $0.76_{-0.01}^{+0.01}$ & $0.491_{-0.02}^{+0.032}$ & $6_{-1}^{+2}$ & $1.68_{-0.24}^{+0.40}$ & $0.07_{-0.01}^{+0.01}$ & $2_{-1}^{+2}$ \\
NGC 5044 & 0.0090 & ${ }^{d} 1.07_{-0.01}^{+0.01}$ & $0.524_{-0.003}^{+0.002}$ & $11_{-0}^{+0}$ & $3.45_{-0.03}^{+0.03}$ & $0.05_{-0.00}^{+0.00}$ & $28_{-1}^{+1}$ \\
NGC 507 & 0.0165 & ${ }^{d} 1.26_{-0.07}^{+0.07}$ & $0.444_{-0.05}^{+0.005}$ & $19_{-1}^{+1}$ & $1.16_{-0.04}^{+0.04}$ & $0.22_{-0.01}^{+0.01}$ & $14_{-2}^{+2}$ \\
S1101 & 0.0580 & ${ }^{c} 3.00_{-0.70}^{+1.20}$ & $0.639_{-0.007}^{+0.006}$ & $55_{-1}^{+1}$ & $2.90_{-0.18}^{+0.19}$ & $0.20_{-0.04}^{+0.06}$ & $299_{-11}^{+179}$ \\
ZwCl 1215 & 0.0750 & $\left(5.58_{-0.78}^{+0.89}\right)$ & $0.819_{-0.034}^{+0.038}$ & $431_{-25}^{+27}$ & $0.27_{-0.01}^{+0.01}$ & $3.00_{-0.33}^{+0.36}$ & $0_{-0}^{+0}$ \\
\hline
\end{tabular}

Note: ${ }^{a}$ Markevitch (1998). ${ }^{b}$ White (2000). ${ }^{c}$ Edge \& Stewart (1991). $T_{\mathrm{m}}$ with a, b or c has no cooling flow correction. ${ }^{d}$ Fukazawa et al. (1998) with cooling flow correction. $T_{\mathrm{m}}$ in a bracket is estimated from the $L_{\mathrm{X}}-T$ relation given by Markevitch (1998). Others are from Reiprich \& Böhringer (2002) and references therein.

Table 2. Cluster properties of the extended cluster sample.

\begin{tabular}{|c|c|c|c|c|c|c|c|}
\hline Name & $z$ & $T_{\mathrm{m}}(\mathrm{keV})$ & $\beta$ & $r_{\mathrm{c}}(\mathrm{kpc})$ & $n_{\text {center }}\left(10^{-2} \mathrm{~cm}^{-3}\right)$ & $t_{\text {cool }}\left(10^{10} \mathrm{yr}\right)$ & $\dot{M}\left(M_{\odot} / \mathrm{yr}\right)$ \\
\hline $3 \mathrm{C} 129$ & 0.0223 & ${ }^{c} 5.60_{-0.60}^{+0.70}$ & $0.601_{-0.131}^{+0.260}$ & $318_{-107}^{+178}$ & $0.18_{-0.04}^{+0.07}$ & $4.71_{-1.39}^{+1.62}$ & $0^{+0}$ \\
\hline A0539 & 0.0288 & $\begin{array}{l}d \\
d .24^{+0.09}\end{array}$ & $0.561^{+0.020}$ & $\begin{array}{r}-107 \\
147^{+13}\end{array}$ & $0.72^{-0.042}$ & $0.84^{+0.13}$ & $3^{+1}$ \\
\hline A0548e & 0.0410 & b $3.10^{+0.109}$ & $0.480^{+0.013}$ & $118^{-12}$ & $0.28^{-0.09}+02$ & $2.09^{-0.11}$ & $0^{-1}$ \\
\hline A0548w & 0.0424 & $\left(1.20_{-0.17}^{+0.10}\right)$ & $0.666_{-0111}^{+0.013}$ & $198_{-61}^{+89}$ & $0.10^{+0.01}$ & $2.28^{-0.67}$ & $0_{-0}^{+0}$ \\
\hline A0644 & 0.0704 & $a_{7.10_{-0.60}^{+0.60}}$ & $0.700_{-0.011}^{+0.0111}$ & $202_{-6}^{+6}$ & $0.78^{+0.01}$ & $\begin{array}{l}2.28^{-0.59} \\
1.18^{+0.08}\end{array}$ & $16^{+29}$ \\
\hline A1413 & 0.1427 & ${ }^{b} 7.32^{+0.26}$ & $0.660^{+0.017}$ & $178^{+12}$ & $1.24^{+0.001}$ & $0.72^{-0.008}$ & $\begin{array}{l}190_{-32}^{+40} \\
19\end{array}$ \\
\hline A 1689 & 0.1840 & $b_{9} 9.23_{-028}^{+0.24}$ & $0.690_{-0.011}^{+0.815}$ & $\begin{array}{l}162^{+6} \\
16\end{array}$ & $\begin{array}{l}1.4-0.07 \\
2.12^{+0.18}\end{array}$ & $0.47^{+0.04}$ & $683^{-323}$ \\
\hline A 1775 & 0.0757 & ${ }^{b} 3.69^{+0.20}$ & $0.673^{+0.026}$ & $259^{+19}$ & $0.30^{+0.05}$ & $\begin{array}{l}0.16^{-0.04} \\
2.16^{+0.36}\end{array}$ & $\begin{array}{c}0_{-18} \\
0^{+0}\end{array}$ \\
\hline A 1800 & 0.0748 & $\left(4.02_{-0.56}^{+0.111}\right)$ & $0.766_{-0.139}^{+0.308}$ & $391_{-131}^{+223}$ & $\begin{array}{l}-0.04 \\
0.18_{-0.04}^{+0.07}\end{array}$ & $3.65_{-1.07}^{+1.14}$ & $0_{-0}^{+0}$ \\
\hline A1914 & 0.1712 & $b 10.53^{-0.56}$ & $\begin{array}{l}0.751_{-0.018}^{+0.139} \\
0.718\end{array}$ & $\begin{array}{l}591-131 \\
230^{+10}\end{array}$ & $\begin{array}{l}0.10-0.04 \\
1.12^{+0.03}\end{array}$ & $\begin{array}{l}0.0 J_{-1.07}^{+0.04} \\
0.97^{+0.04}\end{array}$ & $180^{+50}$ \\
\hline A2151w & 0.0369 & ${ }^{b} 2.40^{+0.06}$ & $0.564^{+0.814}$ & $68^{-10}$ & $0.82^{+0.003}$ & $0.60^{+0.003}$ & $30^{-40}$ \\
\hline A2319 & 0.0564 & ${ }^{a} 9.20_{-0.0}^{+0.96}$ & $0.591_{-0.012}^{+0.013}$ & $284_{-13}^{+14}$ & $0.51^{+0.035}$ & $2.10^{+0.24}$ & $0_{-0}^{+3}$ \\
\hline A2734 & 0.0620 & $\left(3.85^{+0.70}+62\right)$ & $0.624^{+0.034}$ & $211^{-13}+26$ & $0.32^{+0.02}$ & $2.04_{-0.028}^{+0.21}$ & $\begin{array}{l}-0 \\
0^{+0}\end{array}$ \\
\hline A2877 & 0.0241 & $3.50^{+2.20}$ & $0.566^{+0.029}$ & $189^{+18}$ & $\begin{array}{l}.0 .0 .02 \\
0.19^{+0.02}\end{array}$ & $3.33^{+1.60}$ & $\begin{array}{l}-0 \\
0^{+0}\end{array}$ \\
\hline A3395n & 0.0498 & a $4.80^{+0.40}$ & $0.981^{+0.619}$ & $\begin{array}{l}1072^{-16} \\
67383\end{array}$ & $0.10^{+0.02}$ & $7.33^{+2.40}$ & $\begin{array}{l}-0 \\
0^{+0}\end{array}$ \\
\hline A3528n & 0.0540 & $\begin{array}{l}-0.40 \\
3.40^{+1.66}\end{array}$ & $0.621^{+0.034}$ & $\begin{array}{l}072-203 \\
177^{+16}\end{array}$ & $0.34^{+0.02}$ & $\begin{array}{l}1.81^{-2.03} \\
1.01\end{array}$ & $0^{+0}$ \\
\hline A3528s & 0.0551 & $3.15^{-0.84}$ & $0.463^{+0.013}$ & $\begin{array}{l}11^{-15} \\
100^{+8}\end{array}$ & $0.48^{+0.02}$ & $\begin{array}{l}1.01-0.28 \\
1.21^{+0.26}\end{array}$ & $1^{+0}$ \\
\hline A3530 & 0.0544 & $\begin{array}{r}-0.59 \\
3.89^{+0.27}\end{array}$ & $0.773^{-0.012}$ & $420^{-8}+74$ & $\begin{array}{l}0.02 \\
0.013^{+0.01}\end{array}$ & $\begin{array}{r}-0.19 \\
5.27^{+0.61}\end{array}$ & $0^{+1}$ \\
\hline A3532 & 0.0539 & $4.58^{-0.25}$ & $0653^{-0.085}$ & $281^{-61}+26$ & $0.30^{+0.01}$ & $242^{+0.56}$ & $\begin{array}{l}-0 \\
0^{+0}\end{array}$ \\
\hline A3560 & 0.0495 & $\left(3.16^{-0.51}\right)$ & $0.566^{+0.0293}$ & $255^{+34}$ & $0.17^{+0.05}$ & $3.47^{+0.51}$ & $\begin{array}{l}-0 \\
0^{+0}\end{array}$ \\
\hline A3627 & 0.0163 & ${ }^{b} 6.02^{-0.44}$ & $0.555^{-0.029}$ & $299^{-27}$ & $\begin{array}{l}-0.01 \\
0.19^{+0.02}\end{array}$ & $4.51^{-0.44}$ & $\begin{array}{l}-0 \\
0^{+0}\end{array}$ \\
\hline A3695 & 0.0890 & $\begin{array}{r}(5.0 .08 \\
(5.0 .85\end{array}$ & $0.642^{-0.044}$ & $398^{-49}+253$ & $\begin{array}{l}-0.02 \\
0.20^{+0.08}\end{array}$ & $3.82^{+1.25}$ & $\begin{array}{l}-0 \\
0^{+0}\end{array}$ \\
\hline A3822 & 0.0760 & $\left(4.90^{+0.74}\right)$ & $0.639^{+0.1150}$ & $\begin{array}{r}-149 \\
350^{+159}\end{array}$ & $0.21^{+0.05}$ & $\begin{array}{l}3.02-1.12 \\
3.60^{+0.83}\end{array}$ & $\begin{array}{l}-0 \\
0^{+0}\end{array}$ \\
\hline A 3827 & 0.0980 & $\left(7.08^{+0.69}\right.$ & $\begin{array}{l}-0.093 \\
0.989^{+0.410}\end{array}$ & $\begin{array}{l}-111 \\
593+247\end{array}$ & $\begin{array}{l}0.21-0.03 \\
0.26^{+0.06}\end{array}$ & $\begin{array}{l}3.03^{-0.86} \\
3.48 .86\end{array}$ & $\begin{array}{l}-0 \\
0^{+0}\end{array}$ \\
\hline A3888 & 0.1510 & $\left(8.84^{+0.99}\right)$ & $0.928^{+0.192}$ & $\begin{array}{l}-148 \\
400^{+45}\end{array}$ & $\begin{array}{l}0.22^{-0.05} \\
0.04\end{array}$ & $192^{+0.72}$ & $\begin{array}{l}-0 \\
0^{+0}\end{array}$ \\
\hline A3921 & 0.0936 & $\begin{array}{l}0.07-1.24 \\
b 573^{+0.24}\end{array}$ & $\begin{array}{l}0.0 .066 \\
0762+0.036\end{array}$ & $\begin{array}{l}328^{+39} \\
325\end{array}$ & $0.34^{+0.03}$ & $\begin{array}{l}1.0 .25 \\
2.0 .12\end{array}$ & $\begin{array}{l}-0 \\
0^{+0}\end{array}$ \\
\hline AWM7 & 0.0172 & $d_{3.75^{+0.09}}$ & $0.671^{+0.027}$ & $173^{+17}$ & $0.60^{+0.05}$ & 1.00 .12 & $\begin{array}{l}-0 \\
6^{+3}\end{array}$ \\
\hline HCG 94 & 0.0417 & $3.45^{+0.30}$ & $0.514^{-0.0257}$ & $86^{+4^{5}}$ & $0.70^{+0.052}$ & $\begin{array}{l}1.10-0.89 \\
0.89^{+0.83}\end{array}$ & $\begin{array}{l}-4 \\
6^{+3}\end{array}$ \\
\hline IIZw 108 & 0.0494 & $\begin{array}{l}5.4 J_{-0.03} \\
\left(3.44_{-048}^{+0.55}\right)\end{array}$ & $0.662_{-0.097}^{+0.166}$ & $\begin{array}{l}00_{-3}^{3} \\
365^{+159}\end{array}$ & $0.14^{+0.02}$ & $\begin{array}{l}0.09-0.07 \\
4.50^{+1.06}\end{array}$ & $\begin{array}{l}0-2 \\
0^{+0}\end{array}$ \\
\hline M 49 & 0.0044 & $0.95^{+0.02}$ & $0.592^{+0.0007}$ & $10^{+-105}$ & $1.33_{-0.02}^{-0.02}$ & $0.12^{-1.0 .00}$ & $2_{-0}^{-0}$ \\
\hline NGC 499 & 0.0147 & $0.72_{-0.02}^{+0.01}$ & $0.722_{-0.030}^{+0.0074}$ & $23_{-1}^{+2}$ & $0.94_{-0.09}^{+0.102}$ & $\begin{array}{l}0.12_{-0.01}^{+0.00} \\
0.01\end{array}$ & $\begin{array}{l}-0 \\
11^{+4}\end{array}$ \\
\hline NGC 5813 & 0.0064 & $\left(0.52^{+0.08}\right)$ & $0.766^{+0.179}$ & $24_{-5}^{-1}$ & $0.90^{+0.18}$ & $0.10^{+0.001}$ & $9_{-5}^{+13}$ \\
\hline NGC 5846 & 0.0061 & $0.82^{+0.01}$ & $0.599^{+0.016}$ & $6^{+0}$ & $3.56^{-0.22}$ & $0.04^{-0.020}$ & $2^{-5}+0$ \\
\hline OPHIUCHU & 0.0280 & $10.26^{+0.32}$ & $0.747^{+0.0155}$ & $278^{+23}$ & $0.68^{+0.04}$ & $1.72^{-0.012}$ & $0_{-0}^{+0}$ \\
\hline PERSEUS & 0.0183 & ${ }^{d} 6.79^{+0.12}$ & $0.540^{+0.006}$ & $63^{-21}$ & $3.25^{-0.04}$ & $0.28^{+0.01}$ & $481_{-32}^{-0}$ \\
\hline PKS0745 & 0.1028 & ${ }^{b} 7.21_{-0.11}^{+0.12}$ & $0.608^{+0.0006}$ & $71^{-1}$ & $5.70^{+0.014}$ & $0.16^{-0.01}$ & $1424^{+32}$ \\
\hline RXJ2344 & 0.0786 & $\left(4.73_{-0.66}^{+0.11}\right)$ & $0.807_{-0.030}^{-0.006}$ & $300_{-18}^{+19}$ & $\begin{array}{r}-0.15 \\
0.51^{+0.06}\end{array}$ & $1.43^{-0.00}+24$ & $0^{+1}$ \\
\hline S405 & 0.0613 & $\left(4.21_{-059}^{+0.67}\right)$ & $0.664_{-0133}^{+0.2630}$ & $458_{-158}^{+261}$ & $0.12^{+0.05}$ & $5.67^{-0.82}+85$ & $\begin{array}{l}-0 \\
0^{+0}\end{array}$ \\
\hline S540 & 0.0358 & $\left(2.40_{-034}^{+0.58}\right)$ & $0.641_{-0.051}^{+0.073}$ & $\begin{array}{l}130_{-28}^{+158} \\
138\end{array}$ & $0.40^{+0.03}$ & $1.23^{-1.23}$ & $1^{+9}$ \\
\hline S636 & 0.0116 & $\left(1.18_{-017}^{+0.19}\right)$ & $0.752_{-0.23}^{+0.211}$ & $\begin{array}{l}150_{-28}^{-130} \\
343_{-86}^{+13}\end{array}$ & $0.07^{+0.05}$ & $3.06^{+0.77}$ & $\begin{array}{l}1-1 \\
0^{+0}\end{array}$ \\
\hline TRIANGUL & 0.0510 & $\begin{array}{r}a .50^{+0.70} \\
a^{+0.70}\end{array}$ & $0.610_{-0.010}^{+0.0103}$ & $\begin{array}{l}27 J_{-86}^{+10} \\
-10\end{array}$ & $0.55^{+0.01}$ & $1.98^{+0.12}$ & $\begin{array}{l}-0 \\
0^{+0}\end{array}$ \\
\hline UGC0 3957 & 0.0340 & 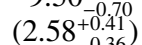 & $0.740^{+0.133}$ & $142^{+44}$ & $0.48^{+0.08}$ & $\begin{array}{l}1.90-0.12 \\
1.09^{+0.21}\end{array}$ & $8^{-0}$ \\
\hline $\mathrm{ZwCl} 1742$ & 0.0757 & $\left(5.23_{-0.73}^{+0.84}\right)$ & $0.717_{-0.053}^{+0.0863}$ & $231_{-38}^{+43}$ & $\begin{array}{l}-0.06 \\
0.60_{-0.09}^{+0.11}\end{array}$ & $1.29_{-0.23}^{+0.20}$ & $0_{-0}^{+83}$ \\
\hline
\end{tabular}

Note: The symbols in $T_{\mathrm{m}}$ have the same meanings as in Table 1 . 
Table 3. Fit parameters with a double $\beta$ model.

\begin{tabular}{|c|c|c|c|c|c|c|c|c|}
\hline Name & $S_{01}\left(10^{-6} \mathrm{cts} / \mathrm{s} / \mathrm{pixel}^{2}\right)$ & $S_{02}\left(10^{-6} \mathrm{cts} / \mathrm{s} / \mathrm{pixel}^{2}\right)$ & $\beta_{1}$ & $\beta_{2}$ & $r_{\mathrm{c} 1}(\mathrm{kpc})$ & $r_{\mathrm{c} 2}(\mathrm{kpc})$ & $\chi_{\mathrm{s}}^{2}$ & $\chi_{\mathrm{d}}^{2}$ \\
\hline A0085 & $27.01 \pm 0.99$ & $1.70 \pm 0.24$ & $0.60 \pm 0.03$ & $0.73 \pm 0.03$ & $58 \pm 3$ & $385 \pm 28$ & 5.32 & 1.90 \\
\hline A0119 & $0.50 \pm 0.03$ & $0.16 \pm 0.03$ & $0.76 \pm 0.11$ & $1.46 \pm 0.24$ & $99 \pm 45$ & $1511 \pm 207$ & 1.75 & 1.47 \\
\hline A0133 & $23.96 \pm 0.82$ & $0.87 \pm 0.06$ & $0.65 \pm 0.02$ & $0.78 \pm 0.02$ & $42 \pm 1$ & $321 \pm 13$ & 4.14 & 1.71 \\
\hline A0401 & $3.93 \pm 0.30$ & $0.51 \pm 0.30$ & $0.69 \pm 0.08$ & $0.66 \pm 0.03$ & $39 \pm 23$ & $525 \pm 93$ & 1.19 & 1.17 \\
\hline A0478 & $37.46 \pm 1.30$ & $4.02 \pm 0.71$ & $0.68 \pm 0.04$ & $0.71 \pm 0.02$ & $72 \pm 4$ & $253 \pm 14$ & 3.53 & 1.50 \\
\hline A0496 & $37.20 \pm 1.87$ & $1.40 \pm 0.33$ & $0.59 \pm 0.05$ & $0.69 \pm 0.04$ & $30 \pm 3$ & $257 \pm 31$ & 3.97 & 1.07 \\
\hline A1367 & $0.30 \pm 0.01$ & $0.22 \pm 0.01$ & $0.96 \pm 0.10$ & $1.51 \pm 0.06$ & $91 \pm 23$ & $976 \pm 31$ & 1.51 & 1.14 \\
\hline A1644 & $1.16 \pm 0.24$ & $0.18 \pm 0.06$ & $0.83 \pm 0.30$ & $2.38 \pm 0.81$ & $74 \pm 92$ & $2169 \pm 532$ & 0.97 & 0.89 \\
\hline A1651 & $7.39 \pm 0.47$ & $2.17 \pm 0.24$ & $0.75 \pm 0.07$ & $0.76 \pm 0.02$ & $20 \pm 11$ & $356 \pm 17$ & 1.46 & 1.10 \\
\hline A1795 & $41.44 \pm 0.60$ & $1.75 \pm 0.16$ & $0.72 \pm 0.02$ & $0.89 \pm 0.02$ & $79 \pm 2$ & $432 \pm 19$ & 10.04 & 1.99 \\
\hline A2029 & $55.67 \pm 1.98$ & $5.81 \pm 1.16$ & $0.63 \pm 0.03$ & $0.65 \pm 0.02$ & $62 \pm 3$ & $213 \pm 15$ & 2.61 & 1.40 \\
\hline A2052 & $23.26 \pm 1.43$ & $3.78 \pm 0.46$ & $2.10 \pm 1.02$ & $0.66 \pm 0.02$ & $84 \pm 25$ & $140 \pm 12$ & 2.70 & 1.45 \\
\hline A2063 & $4.71 \pm 0.00$ & $0.51 \pm 0.00$ & $0.49 \pm 0.00$ & $2.02 \pm 0.00$ & $55 \pm 0$ & $640 \pm 0$ & 1.97 & 1.17 \\
\hline A2142 & $19.59 \pm 0.69$ & $0.86 \pm 0.19$ & $0.67 \pm 0.04$ & $1.01 \pm 0.13$ & $40 \pm 8$ & $893 \pm 130$ & 2.55 & 1.39 \\
\hline A 2255 & $0.35 \pm 0.05$ & $0.56 \pm 0.05$ & $1.15 \pm 0.32$ & $0.90 \pm 0.03$ & $69 \pm 93$ & $778 \pm 31$ & 1.38 & 1.36 \\
\hline A2589 & $3.45 \pm 0.28$ & $0.51 \pm 0.23$ & $0.66 \pm 0.10$ & $0.74 \pm 0.07$ & $95 \pm 13$ & $311 \pm 56$ & 1.38 & 1.23 \\
\hline A2634 & $0.42 \pm 0.04$ & $0.13 \pm 0.01$ & $0.47 \pm 0.03$ & $1.89 \pm 0.18$ & $80 \pm 10$ & $1189 \pm 77$ & 1.77 & 1.25 \\
\hline A2657 & $2.34 \pm 0.00$ & $0.25 \pm 0.00$ & $0.89 \pm 0.00$ & $1.27 \pm 0.00$ & $48 \pm 0$ & $796 \pm 0$ & 3.05 & 1.68 \\
\hline A3112 & $41.34 \pm 2.59$ & $3.11 \pm 1.41$ & $0.63 \pm 0.06$ & $0.62 \pm 0.03$ & $51 \pm 5$ & $164 \pm 24$ & 1.62 & 1.44 \\
\hline A 3266 & $1.24 \pm 0.05$ & $0.76 \pm 0.04$ & $1.20 \pm 0.11$ & $1.27 \pm 0.03$ & $50 \pm 29$ & $1162 \pm 28$ & 2.62 & 1.76 \\
\hline A3391 & $0.87 \pm 0.16$ & $0.66 \pm 0.08$ & $0.50 \pm 0.06$ & $0.66 \pm 0.03$ & $71 \pm 19$ & $335 \pm 24$ & 1.30 & 1.26 \\
\hline A 3526 & $8.97 \pm 0.79$ & $0.34 \pm 0.07$ & $0.57 \pm 0.03$ & $0.70 \pm 0.05$ & $33 \pm 3$ & $272 \pm 32$ & 1.22 & 1.11 \\
\hline A 3558 & $3.13 \pm 0.05$ & $0.20 \pm 0.04$ & $0.68 \pm 0.03$ & $1.17 \pm 0.11$ & $32 \pm 8$ & $1198 \pm 119$ & 3.66 & 2.90 \\
\hline A 3562 & $2.84 \pm 0.10$ & $0.07 \pm 0.03$ & $0.52 \pm 0.02$ & $1.26 \pm 0.27$ & $03 \pm 6$ & $1341 \pm 273$ & 2.10 & 1.78 \\
\hline A3571 & $5.00 \pm 0.40$ & $4.39 \pm 0.22$ & $0.82 \pm 0.13$ & $0.68 \pm 0.01$ & $94 \pm 13$ & $256 \pm 6$ & 1.72 & 1.13 \\
\hline A3667 & $2.10 \pm 0.04$ & $0.20 \pm 0.01$ & $0.89 \pm 0.03$ & $1.70 \pm 0.09$ & $02 \pm 12$ & $2375 \pm 95$ & 2.53 & 1.63 \\
\hline A4038 & $10.37 \pm 0.58$ & $0.49 \pm 0.29$ & $0.58 \pm 0.04$ & $0.70 \pm 0.12$ & $53 \pm 4$ & $241 \pm 69$ & 1.27 & 1.18 \\
\hline A4059 & $8.67 \pm 0.46$ & $0.43 \pm 0.18$ & $0.64 \pm 0.06$ & $0.90 \pm 0.19$ & $82 \pm 7$ & $438 \pm 112$ & 1.32 & 1.06 \\
\hline COMA & $1.13 \pm 0.54$ & $1.35 \pm 0.52$ & $0.57 \pm 0.05$ & $0.90 \pm 0.23$ & $29 \pm 66$ & $444 \pm 104$ & 1.09 & 1.09 \\
\hline HYDRA-A & $40.63 \pm 1.40$ & $5.67 \pm 0.43$ & $1.84 \pm 0.42$ & $0.73 \pm 0.01$ & $98 \pm 14$ & $183 \pm 8$ & 6.24 & 1.81 \\
\hline MKW3S & $12.40 \pm 0.92$ & $3.84 \pm 0.62$ & $1.42 \pm 0.41$ & $0.68 \pm 0.02$ & $91 \pm 17$ & $152 \pm 13$ & 2.07 & 1.54 \\
\hline S1101 & $34.57 \pm 1.35$ & $0.58 \pm 0.30$ & $0.79 \pm 0.08$ & $0.96 \pm 0.16$ & $66 \pm 6$ & $381 \pm 104$ & 1.67 & 1.33 \\
\hline A0539 & $1.53 \pm 0.19$ & $0.41 \pm 0.08$ & $0.53 \pm 0.09$ & $0.75 \pm 0.08$ & $42 \pm 9$ & $313 \pm 40$ & 1.77 & 1.19 \\
\hline A1413 & $10.29 \pm 0.59$ & $1.11 \pm 0.20$ & $0.80 \pm 0.07$ & $0.91 \pm 0.05$ & $55 \pm 12$ & $559 \pm 47$ & 1.73 & 1.45 \\
\hline A1689 & $23.46 \pm 1.39$ & $2.53 \pm 0.82$ & $0.88 \pm 0.14$ & $0.91 \pm 0.05$ & $52 \pm 19$ & $471 \pm 56$ & 1.73 & 1.24 \\
\hline A1775 & $1.48 \pm 0.08$ & $0.18 \pm 0.04$ & $2.05 \pm 0.69$ & $1.70 \pm 0.53$ & $40 \pm 14$ & $1443 \pm 382$ & 1.62 & 1.46 \\
\hline A2319 & $3.59 \pm 0.23$ & $0.76 \pm 0.19$ & $1.06 \pm 0.19$ & $0.82 \pm 0.06$ & $83 \pm 48$ & $874 \pm 120$ & 1.34 & 1.24 \\
\hline A 2877 & $0.28 \pm 0.02$ & $0.15 \pm 0.01$ & $3.58 \pm 0.89$ & $1.23 \pm 0.07$ & $23 \pm 47$ & $606 \pm 28$ & 2.79 & 2.04 \\
\hline A3532 & $0.91 \pm 0.11$ & $0.27 \pm 0.09$ & $0.74 \pm 0.24$ & $1.09 \pm 0.21$ & $93 \pm 48$ & $761 \pm 161$ & 1.31 & 1.12 \\
\hline A3888 & $3.90 \pm 0.22$ & $0.30 \pm 0.15$ & $1.39 \pm 0.19$ & $1.71 \pm 0.39$ & $03 \pm 47$ & $1341 \pm 271$ & 0.90 & 0.90 \\
\hline AWM7 & $2.53 \pm 0.22$ & $0.56 \pm 0.11$ & $0.78 \pm 0.11$ & $0.88 \pm 0.05$ & $25 \pm 16$ & $406 \pm 39$ & 1.40 & 1.33 \\
\hline HCG 94 & $2.26 \pm 0.09$ & $0.41 \pm 0.04$ & $0.53 \pm 0.01$ & $0.58 \pm 0.01$ & $59 \pm 3$ & $199 \pm 10$ & 2.43 & 2.20 \\
\hline NGC 507 & $1.20 \pm 0.30$ & $3.37 \pm 0.34$ & $0.76 \pm 0.04$ & $4.29 \pm 1.21$ & $41 \pm 4$ & $73 \pm 11$ & 2.41 & 2.40 \\
\hline NGC 5846 & $10.88 \pm 0.97$ & $1.48 \pm 0.18$ & $0.51 \pm 0.01$ & $4.78 \pm 1.07$ & $3 \pm 0$ & $55 \pm 7$ & 1.78 & 1.53 \\
\hline OPHIUCHU & $4.86 \pm 0.24$ & $0.35 \pm 0.10$ & $1.04 \pm 0.11$ & $1.40 \pm 0.22$ & $28 \pm 26$ & $1190 \pm 177$ & 1.41 & 1.37 \\
\hline PKS 0745 & $53.06 \pm 1.73$ & $2.02 \pm 0.39$ & $0.70 \pm 0.02$ & $0.65 \pm 0.01$ & $72 \pm 2$ & $235 \pm 16$ & 1.17 & 1.06 \\
\hline RXJ 2344 & $1.41 \pm 0.20$ & $1.41 \pm 0.14$ & $0.72 \pm 0.13$ & $0.92 \pm 0.04$ & $28 \pm 27$ & $400 \pm 20$ & 1.76 & 1.70 \\
\hline TRIANGUL & $3.36 \pm 0.09$ & $0.66 \pm 0.06$ & $0.71 \pm 0.03$ & $0.80 \pm 0.02$ & $48 \pm 9$ & $700 \pm 30$ & 1.57 & 1.28 \\
\hline $\mathrm{ZwCl} 1742$ & $3.50 \pm 0.57$ & $0.58 \pm 0.33$ & $0.83 \pm 0.21$ & $0.93 \pm 0.16$ & $94 \pm 45$ & $547 \pm 135$ & 1.11 & 1.11 \\
\hline
\end{tabular}

Note: $\chi_{\mathrm{s}}^{2}$ and $\chi_{\mathrm{d}}^{2}$ are the reduced $\chi^{2}$ values for the single $\beta$ model and the double $\beta$ model, respectively. $S_{1}$ and $S_{2}$ are the surface brightness. Note $1^{\prime}=120$ pixels. 
Y. Chen et al.: Statistics of X-ray observables of galaxy clusters, Online Material p 5

Table 4. Cluster properties for the scaling relations.

\begin{tabular}{|c|c|c|c|c|c|c|c|}
\hline Name & $\begin{array}{c}T_{\mathrm{h}} \\
(\mathrm{keV})\end{array}$ & $\begin{array}{c}L_{\mathrm{X}} \\
\left(10^{44} \mathrm{erg} / \mathrm{s}, 0.1-2.4 \mathrm{keV}\right)\end{array}$ & $\begin{array}{c}M_{500} \\
\left(10^{14} M_{\odot}\right)\end{array}$ & $\begin{array}{c}r_{500} \\
(\mathrm{Mpc})\end{array}$ & $f_{\mathrm{gas}}$ & $\begin{array}{c}M_{\text {gas, } 500} \\
\left(10^{13} M_{\odot}\right)\end{array}$ & $\begin{array}{c}L_{\mathrm{nir}} \\
\left(h_{70}^{-2} \times 10^{12} L_{\odot}\right)\end{array}$ \\
\hline $2 \mathrm{~A} 0335$ & $3.64_{-0.08}^{+0.09}$ & $4.64 \pm 0.04$ & $2.79_{-1.63}^{+1.09}$ & $1.20_{-0.30}^{+0.14}$ & $0.19_{-0.03}^{+0.13}$ & $5.42_{-1.74}^{+0.80}$ & $3.44 \pm 0.07$ \\
\hline A0085 & $6.51_{-0.23}^{+0.16}$ & $9.67 \pm 0.11$ & $8.08_{-3.51}^{+1.55}$ & $1.68_{-0.29}^{+0.10}$ & $0.21_{-0.02}^{+0.09}$ & $16.66_{-354}^{+1.49}$ & $5.99 \pm 0.06$ \\
\hline A0119 & $5.69_{-0.28}^{+0.23}$ & $3.34 \pm 0.05$ & $8.98_{-2.59}^{+1.51}$ & $\begin{array}{l}-0.29 \\
1.76_{-0.19}^{+0.07}\end{array}$ & $0.15_{-0.01}^{+0.02}$ & $13.24_{-179}^{+0.54}$ & $6.46 \pm 0.07$ \\
\hline A0133 & $3.97_{-0.27}^{-0.28}$ & $2.85 \pm 0.04$ & $\begin{array}{r}-2.59 \\
4.30_{-2.08}^{+1.00}\end{array}$ & $1.36_{-0.27}^{+0.19}$ & $0.14_{-0.02}^{-0.01}$ & $6.01_{-1.36}^{+0.51}$ & $3.72 \pm 0.04$ \\
\hline A0262 & $2.25_{-0.06}^{+0.06}$ & $0.98 \pm 0.06$ & $0.94_{-0.12}^{+0.05}$ & $0.85_{-0.04}^{+0.02}$ & $0.21_{-0.05}^{+0.05}$ & $1.93_{-0.48}^{+0.39}$ & $2.07 \pm 0.07$ \\
\hline A0399 & $6.46_{-0.36}^{+0.30}$ & $7.13 \pm 0.62$ & $7.74_{-2.32}^{+2.15}$ & $1.63_{-0.18}^{+0.14}$ & $0.19_{-0.07}^{+0.11}$ & $14.79_{-5.62}^{+3.43}$ & - \\
\hline A0400 & $2.43_{-0.12}^{+0.13}$ & $0.65 \pm 0.01$ & $1.33_{-0.16}^{+0.11}$ & $0.95_{-0.04}^{+0.02}$ & $0.16_{-0.01}^{+0.02}$ & $2.11_{-0.20}^{+0.10^{2}}$ & $2.34 \pm 0.06$ \\
\hline A0401 & $7.19_{-0.24}^{+0.28}$ & $12.41 \pm 0.22$ & $8.38_{-2.84}^{+0.16}$ & $1.67_{-0.021}^{+0.04}$ & $0.25_{-0.02}^{+0.08}$ & $20.55_{-4.27}^{+2.75}$ & - \\
\hline A0478 & $6.91_{-0.036}^{+0.40}$ & $17.44 \pm 0.18$ & $8.85_{-4.69}^{+3.84}$ & $1.68_{-0.037}^{+0.11}$ & $0.22_{-0.04}^{+0.14}$ & $19.10_{-4.42}^{+2.18}$ & $7.96 \pm 0.09$ \\
\hline A0496 & $4.59_{-0.10}^{+0.10}$ & $3.77 \pm 0.05$ & $4.81_{-2.11}^{+0.89}$ & $1.44_{-0.025}^{+0.08}$ & $0.16_{-0.02}^{+0.08}$ & $7.77_{-1.65}^{+0.77^{2}}$ & $3.91 \pm 0.05$ \\
\hline A0576 & $3.83_{-0.15}^{+0.10}$ & $1.86 \pm 0.21$ & $4.61_{-2.39}^{+3.25}$ & $1.42_{-0.03}^{+0.28}$ & $0.12_{-0.08}^{+0.18}$ & $5.43^{-2.153}$ & $4.62 \pm 0.06$ \\
\hline A0754 & $9.00_{-0.34}^{+0.35}$ & $3.97 \pm 0.11$ & $13.86_{-6.43}^{+4.40}$ & $2.02_{-0.38}^{+0.19}$ & $0.10_{-0.02}^{+0.08}$ & $13.25_{-3.32}^{+1.83}$ & $9.28 \pm 0.05$ \\
\hline A1060 & $3.15_{-0.05}^{+0.05}$ & $0.56 \pm 0.03$ & $2.50_{-1.02}^{+0.62}$ & $\begin{array}{l}1.19_{-0.09}^{+0.09} \\
\end{array}$ & $0.09_{-0.02}^{+0.04}$ & $2.19^{+0.49}$ & $2.39 \pm 0.06$ \\
\hline A1367 & $3.55_{-0.08}^{+0.08}$ & $1.20 \pm 0.02$ & $7.42_{-2.11}^{+1.11}$ & $1.69_{-0.08}^{+0.08}$ & $0.09_{-0.01}^{+0.03}$ & $6.39_{-0.30}^{+0.69}$ & $3.81 \pm 0.05$ \\
\hline A1644 & ${ }^{a} 4.70_{-0.70}^{+0.90}$ & $3.92 \pm 0.34$ & $7.34_{-4.40}^{+4.30}$ & $1.64_{-0.43}^{+0.20}$ & $0.17_{-0.04}^{-0.01}$ & $12.53_{-428}^{+2.71}$ & $5.82 \pm 0.05$ \\
\hline A 1650 & $5.68_{-0.27}^{+0.30}$ & $7.33 \pm 0.79$ & $6.53^{-4.417}$ & $1.52_{-0 ? 2}^{+0.43}$ & $0.19_{-0.08}^{+0.04}$ & $12.22_{-579}^{+4.85}$ & $3.09 \pm 0.14$ \\
\hline A 1651 & $6.22_{-0.41}^{-0.27}$ & $7.85 \pm 0.14$ & $8.29_{-3.60}^{+2.43}$ & $1.65_{-0.28}^{+0.122}$ & $0.17_{-0.02}^{+0.08}$ & $14.38_{-2.81}^{+1.79}$ & $7.82 \pm 0.05$ \\
\hline A1736 & $3.68_{-0.17}^{+0.41}$ & $3.22 \pm 0.33$ & $2.17_{-0.69}^{+3.60}$ & $1.09_{-0.13}^{+0.18}$ & $0.25_{-0.10}^{+0.82}$ & $5.33_{-1.84}^{+1.081}$ & $5.28 \pm 0.10$ \\
\hline A1795 & $6.17_{-0.25}^{+0.26}$ & $10.00 \pm 0.07$ & $9.87_{-5.88}^{+3.69}$ & $1.79_{-0.21}^{+0.21}$ & $0.15_{-0.03}^{+0.11}$ & $14.51_{-3.16}^{+1.54}$ & $4.80 \pm 0.04$ \\
\hline A2029 & $7.93_{-0.36}^{+0.39}$ & $17.07 \pm 0.18$ & $9.95_{-5.36}^{+3.48}$ & $1.77_{-0.18}^{+0.18}$ & $0.21_{-0.03}^{+0.12}$ & $20.92_{-552}^{+3.45}$ & $8.12 \pm 0.06$ \\
\hline A2052 & $3.12_{-0.09}^{-0.36}$ & $2.37 \pm 0.04$ & $\begin{array}{r}-5.16 \\
2.70_{-1.32}^{+0.57}\end{array}$ & $1.19_{-0.024}^{+0.08}$ & $\begin{array}{r}-0.03 \\
0.16_{-0.02}^{+0.09}\end{array}$ & $4.31_{-0.95}^{+1.53^{+1}}$ & $3.22 \pm 0.05$ \\
\hline A2063 & $\begin{array}{r}-0.09 \\
3.56_{-0.12}^{+0.16}\end{array}$ & $2.26 \pm 0.05$ & $2.36_{-0.19}^{+0.32}$ & $1.14_{-0.010}^{-0.24}$ & $0.19_{-0.01}^{+0.02}$ & $4.44_{-0.52}^{+0.95}$ & $3.50 \pm 0.04$ \\
\hline A2065 & $5.37_{-0.30}^{-0.12}$ & $5.63 \pm 0.55$ & $11.19_{-6.52}^{+9.59}$ & $1.84_{-0.50}^{+0.42}$ & $0.12_{-0.08}^{-0.01}$ & $13.68_{-9.46}^{+5.07}$ & $7.33 \pm 0.05$ \\
\hline A 2142 & $8.46_{-0.49}^{+0.53}$ & $21.05 \pm 0.29$ & $14.33_{-6,83}^{+3.62}$ & $1.97_{-0.38}^{+0.15}$ & $0.22_{-0.03}^{+0.11}$ & $31.94_{-7.17}^{+3.46}$ & $7.20 \pm 0.09$ \\
\hline A 2147 & $4.34_{-0.13}^{+0.12}$ & $2.87 \pm 0.15$ & $2.31_{-0.36}^{+0.48}$ & $1.13_{-0.06}^{+0.06}$ & $0.26_{-0.08}^{+0.03}$ & $5.93_{-166}^{+1.19}$ & $4.15 \pm 0.05$ \\
\hline A2163 & $10.55_{-0.68}^{-0.13}$ & $32.16 \pm 0.82$ & $16.00_{-4.86}^{+3.48}$ & $1.85_{-0.21}^{+0.06}$ & $0.27_{-0.03}^{+0.08}$ & $43.75_{-7.16}^{-1.66}$ & - \\
\hline A2199 & $4.28_{-0.10}^{+0.68}$ & $4.20 \pm 0.12$ & $4.29_{-189}^{-1.186}$ & $1.39_{-0.02}^{+0.12}$ & $0.17_{-0.03}^{+0.03}$ & $7.35_{-173}^{-0.916}$ & $4.43 \pm 0.04$ \\
\hline A2204 & $6.38_{-0.23}^{+0.10}$ & $25.89 \pm 0.69$ & $5.82_{-2.98}^{+1.89}$ & $1.38_{-020}^{+0.14}$ & $0.29_{-0.05}^{+0.03}$ & $\begin{array}{r}16.85_{-470}^{+2.24} \\
16\end{array}$ & $\begin{array}{c}- \\
-\end{array}$ \\
\hline A2244 & $5.77^{-0.61}$ & $8.30 \pm 0.28$ & $5.48^{+1.48}$ & $1.42^{+0.12}$ & $0.21^{+0.05}$ & $11.52_{-265}^{+4.70}$ & - \\
\hline A2255 & $5.92_{-026}^{+0.40}$ & $5.46 \pm 0.11$ & $\begin{array}{l}7.40_{-2.23}^{+0.92} \\
7.86_{-167}^{0.92}\end{array}$ & $1.63_{-012}^{+0.06}$ & $0.19_{-0.01}^{+0.04}$ & $14.66_{-150}^{+1.65}$ & $8.70 \pm 0.08$ \\
\hline A2256 & $6.83^{+0.23}$ & $9.24 \pm 0.22$ & $12.12^{-3.67} .41$ & $1.91^{+0.16}$ & $0.18^{-0.01}$ & $\begin{array}{r}-1.50 \\
21.78^{+2.19}\end{array}$ & $10.11 \pm 0.04$ \\
\hline A2589 & $3.38_{-0.13}^{+0.21}$ & $1.87 \pm 0.04$ & $3.24_{-140}^{+0.54}$ & $1.26^{+0.025}$ & $0.15^{-0.03}$ & $\begin{array}{l}4.76^{+0.81}+09 \\
400\end{array}$ & $2.51 \pm 0.06$ \\
\hline A2597 & $\begin{array}{r}-0.13 \\
4.20^{+0.49}\end{array}$ & $6.75 \pm 0.14$ & $3.71^{-1.40}$ & $1.26^{+0.14}$ & $0.17^{-0.02}$ & $\begin{array}{r}-0.99 \\
6.48^{+0.86}\end{array}$ & - \\
\hline A2634 & $3.45^{-0.16}$ & $0.99 \pm 0.03$ & $4.51^{-2.22}$ & $1.42^{+0.033}$ & $0.11^{+0.003}$ & $5.10^{+0.02}$ & $4.53 \pm 0.08$ \\
\hline A2657 & $3.53^{+0.12}$ & $1.74 \pm 0.03$ & $6.06^{+1.00}$ & $1.55^{+0.11}$ & $\begin{array}{l}-0.01 \\
0.10^{+0.05}\end{array}$ & $6.26^{+0.39}$ & $2.35 \pm 0.09$ \\
\hline A 3112 & $4.72^{+0.37}$ & $7.32 \pm 0.15$ & $4.36^{-1.22}$ & $1.34^{+0.26}$ & $0.20^{+0.13}$ & $8.87^{-1.08}$ & $4.11 \pm 0.08$ \\
\hline A3158 & $5.41^{-0.25}$ & $5.61 \pm 0.15$ & $5.75^{-0.89}$ & $1.49^{+0.09}$ & $0.20^{+0.03}$ & $11.63^{-2.1 .13}$ & $6.76 \pm 0.05$ \\
\hline A 3266 & $7.72^{-0.34}$ & $8.62 \pm 0.11$ & $19.24^{-1.4 .76}$ & $2.23^{-0.16}$ & $0.14^{-0.03}$ & $27.35^{-1.99}$ & $7.79 \pm 0.06$ \\
\hline A3376 & $\begin{array}{r}4.43^{-0.38} \\
4.0 .39\end{array}$ & $2.16 \pm 0.05$ & $6.77^{-1.55}$ & $1.60^{-0.34}$ & $0.13^{-0.02}$ & $8.70^{+0.86}$ & $3.81 \pm 0.04$ \\
\hline A3391 & $5.89_{-0.033}^{+0.48}$ & $2.64 \pm 0.08$ & $6.04_{-169}^{+0.99}$ & $\begin{array}{l}1.53_{-0.07}^{+0.06} \\
1.06\end{array}$ & $0.14_{-0.01}^{+0.02}$ & $8.63_{-111}^{+0.50}$ & $5.84 \pm 0.09$ \\
\hline A3395s & $5.55_{-0.65}^{+0.89}$ & $2.12 \pm 0.13$ & $9.48_{-429}^{+4.39}$ & $1.78_{-0.03}^{+0.16}$ & $0.10_{-0.04}^{+0.09}$ & $9.39^{+2.49}$ & $5.61 \pm 0.06$ \\
\hline A3526 & $3.69_{-0.05}^{+0.05}$ & $1.19 \pm 0.04$ & $3.41_{-136}^{+0.60}$ & $1.32_{-020}^{+0.07}$ & $0.11_{-0.01}^{+0.04}$ & $3.83_{-079}^{+0.39}$ & $3.66 \pm 0.16$ \\
\hline A3558 & $5.37_{-0.15}^{+0.17}$ & $6.56 \pm 0.04$ & $6.71_{-212}^{+0.91}$ & $1.59_{-0.07}^{+0.07}$ & $0.22_{-0.02}^{+0.05}$ & $14.60^{+0.85}$ & $11.10 \pm 0.06$ \\
\hline A3562 & $4.47_{-0.21}^{+0.23}$ & $3.08 \pm 0.05$ & $3.51_{-0.43}^{+0.43}$ & $1.28_{-012}^{+0.05}$ & $0.20_{-0.02}^{+0.82}$ & $7.07_{-110}^{-0.50}$ & $3.23 \pm 0.08$ \\
\hline A3571 & $6.80_{-0.18}^{+0.21}$ & $8.08 \pm 0.11$ & $8.76_{-3.43}^{+1.91}$ & $1.75_{-0.27}^{+0.12}$ & $0.19_{-0.02}^{-0.02}$ & $16.45_{-2.93}^{+1.36}$ & - \\
\hline A 3581 & $1.83_{-0.02}^{-0.18}$ & $0.60 \pm 0.03$ & $0.93_{-0.38}^{+0.13}$ & $0.84_{-0.14}^{-0.27}$ & $0.15_{-0.03}^{+0.02}$ & $1.41_{-0.41}^{+0.293}$ & $1.22 \pm 0.09$ \\
\hline A3667 & $6.28_{-0.26}^{+0.27}$ & $9.48 \pm 0.11$ & $5.28_{-1.52}^{+0.52}$ & $1.46_{-0.05}^{+0.05}$ & $0.30_{-0.03}^{+0.03}$ & $16.04_{-2.22}^{+1.15}$ & $8.65 \pm 0.06$ \\
\hline A4038 & $3.22_{-0.10}^{+0.16}$ & $1.92 \pm 0.04$ & $2.58_{-1.05}^{+0.49}$ & $1.18_{-0.19}^{+0.01}$ & $0.16_{-0.03}^{+0.08}$ & $4.14_{-0.92}^{+0.21^{2}}$ & $2.85 \pm 0.06$ \\
\hline A4059 & $3.94_{-0.15}^{+0.10}$ & $2.80 \pm 0.06$ & $4.41_{-2.03}^{+1.05}$ & $1.39_{-0.026}^{+0.19}$ & $0.14_{-0.02}^{+0.03}$ & $\begin{array}{r}-0.92 \\
6.17_{-1.40}^{+0.79}\end{array}$ & $3.12 \pm 0.10$ \\
\hline COMA & $8.07^{+0.29}$ & $8.09 \pm 0.19$ & $9.95_{-2.99}^{+2.03}$ & $1.86_{-0.2}^{+0.26}$ & $0.19_{-0.07}^{+0.02}$ & $19.00_{-3.69}^{+1.89}$ & $8.94 \pm 0.05$ \\
\hline EXO0422 & ${ }^{a} 2.90_{-0.60}^{+0.27}$ & $2.03 \pm 0.21$ & $2.72_{-1.25}^{+1.99}$ & $1.19_{-027}^{+0.21}$ & $0.14_{-0.06}^{-0.04}$ & $3.68_{-175}^{+2.04}$ & $1.63 \pm 0.08$ \\
\hline FORNAX & $1.56_{-0.07}^{+0.050}$ & $0.08 \pm 0.01$ & $1.29_{-0.55}^{+0.45}$ & $0.96^{+0.10}$ & $0.04^{+0.002}$ & $0.46^{+0.15}$ & $\begin{array}{c}1.00+00 \\
-\end{array}$ \\
\hline HYDRA-A & $3.82_{-0.0 .07}^{+0.20}$ & $5.84 \pm 0.04$ & $4.07_{-2.14}^{+0.55}$ & $1.34^{+0.16}$ & $0.18^{+0.11}$ & $\begin{array}{l}0.0 .14 \\
7.29^{+0.17}\end{array}$ & $3.09 \pm 0.02$ \\
\hline IIIZw54 & $\left(3.00_{-0.95}^{+1.317}\right)$ & $0.83 \pm 0.10$ & $3.76_{-2.26}^{+2.14}$ & $1.33_{-0.35}^{+0.29}$ & $0.08_{-0.05}^{+0.03}$ & $2.95^{-1.97}$ & $\begin{array}{c}.0702 \\
-\end{array}$ \\
\hline MKW3S & $3.45_{-0.10}^{+0.95}$ & $2.79 \pm 0.05$ & $3.22_{-153}^{+0.26}$ & $1.25_{-024}^{+0.11}$ & $0.15_{-0.02}^{+0.05}$ & $4.92_{-1.106}^{+0.72}$ & $1.96 \pm 0.08$ \\
\hline MKW4 & $1.84_{-0.03}^{+0.05}$ & $0.34 \pm 0.01$ & $0.69_{-014}^{+0.53}$ & $0.76_{-0.06}^{+0.04}$ & $0.15_{-0.01}^{+0.02}$ & $1.05_{-015}^{+0.06}$ & $1.71 \pm 0.23$ \\
\hline MKW8 & $3.29_{-0.22}^{+0.03}$ & $0.79 \pm 0.11$ & $2.00_{-0.59}^{+0.44}$ & $1.08_{-0.12}^{+0.06}$ & $0.11_{-0.06}^{+0.08}$ & $2.22_{-1.29}^{+1.11}$ & $2.13 \pm 0.08$ \\
\hline NGC 1550 & $1.44_{-0.02}^{-0.22}$ & $0.28 \pm 0.02$ & $0.68_{-0.24}^{+0.59}$ & $0.77_{-0.010}^{+0.12}$ & $0.12_{-0.04}^{+0.06}$ & $\begin{array}{l}-1.29 \\
0.79_{-0.33}^{+0.29}\end{array}$ & - \\
\hline NG 4636 & $0.66_{-0.01}^{-0.82}$ & $0.02 \pm 0.00$ & $0.18_{-0.06}^{+0.24}$ & $0.49_{-0.07}^{+0.03}$ & $0.04_{-0.02}^{+0.04}$ & $0.08_{-0.04}^{+0.33}$ & - \\
\hline
\end{tabular}


Table 4. continued.

\begin{tabular}{cccccccc}
\hline \hline Name & $\begin{array}{c}T_{\mathrm{h}} \\
(\mathrm{keV})\end{array}$ & $\begin{array}{c}L_{\mathrm{X}} \\
\left(10^{44} \mathrm{erg} / \mathrm{s}, 0.1-2.4 \mathrm{keV}\right)\end{array}$ & $\begin{array}{c}M_{500} \\
\left(10^{14} M_{\odot}\right)\end{array}$ & $\begin{array}{c}r_{500} \\
(\mathrm{Mpc})\end{array}$ & $f_{\text {gas }}$ & $\begin{array}{c}M_{\text {gas,500 }} \\
\left(10^{13} M_{\odot}\right)\end{array}$ & $\begin{array}{c}L_{\text {nir }} \\
\left(h_{70}^{-2} \times 10^{12}\right.\end{array}$ \\
\hline NGC 5044 & $1.22_{-0.04}^{+0.04}$ & $0.18 \pm 0.00$ & $0.49_{-0.25}^{+0.12}$ & $0.69_{-0.15}^{+0.05}$ & $0.09_{-0.01}^{+0.04}$ & $0.45_{-0.14}^{+0.05}$ & - \\
NGC 507 & $1.40_{-0.07}^{+0.04}$ & $0.23 \pm 0.00$ & $0.46_{-0.07}^{+0.02}$ & $0.67_{-0.04}^{+0.01}$ & $0.15_{-0.01}^{+0.02}$ & $0.68_{-0.08}^{+0.04}$ & $1.94 \pm 0.16$ \\
S1101 & $2.60_{-0.50}^{+0.50}$ & $3.52 \pm 0.05$ & $2.94_{-1.88}^{+1.57}$ & $1.20_{-0.34}^{+0.18}$ & $0.14_{-0.04}^{+0.15}$ & $4.22_{-1.23}^{+0.90}$ & $2.18 \pm 0.08$ \\
ZwCl 1215 & $\left(6.36_{-2.01}^{+2.94}\right)$ & $5.17 \pm 0.11$ & $9.46_{-4.87}^{+5.94}$ & $1.74_{-0.37}^{+0.30}$ & $0.15_{-0.04}^{+0.88}$ & $13.87_{-3.76}^{+3.01}$ & - \\
\hline
\end{tabular}

Note: The values of $T_{\mathrm{h}}$ and $L_{\mathrm{X}}$ are from Ikebe et al. (2002). $T_{\mathrm{h}}$ in a bracket is estimated from the $L_{\mathrm{X}}-T$ relation (Ikebe et al. 2002). $T_{\mathrm{m}}$ with "a" is derived from non-ASCA spectroscopy (Ikebe et al. 2002, and references therein).

Table 5. Cluster properties for the scaling relations of the extended cluster sample.

\begin{tabular}{|c|c|c|c|c|c|c|c|}
\hline Name & $\begin{array}{c}T_{\mathrm{h}} \\
(\mathrm{keV})\end{array}$ & $\begin{array}{c}L_{\mathrm{X}} \\
\left(10^{44} \mathrm{erg} / \mathrm{s}, 0.1-2.4 \mathrm{keV}\right)\end{array}$ & $\begin{array}{c}M_{500} \\
\left(10^{14} M_{\odot}\right)\end{array}$ & $\begin{array}{c}r_{500} \\
(\mathrm{Mpc})\end{array}$ & $f_{\text {gas }}$ & $\begin{array}{c}M_{\text {gas, } 500} \\
\left(10^{13} M_{\odot}\right)\end{array}$ & $\begin{array}{c}L_{\text {nir }} \\
\left(h_{70}^{-2} \times 10^{12} L_{\odot}\right)\end{array}$ \\
\hline $3 \mathrm{C} 129$ & $5.57_{-0.15}^{+0.16}$ & $2.27 \pm 0.21$ & $5.39_{-2.33}^{+2.26}$ & $1.51_{-0.26}^{+0.19}$ & $0.16_{-0.10}^{+0.16}$ & $8.55_{-4.82}^{+2.58}$ & - \\
\hline A0539 & $3.04_{-0.10}^{+0.11}$ & $1.11 \pm 0.02$ & $2.68_{-0.85}^{+0.33}$ & $1.19_{-0.14}^{+0.26}$ & $0.14_{-0.01}^{+0.10}$ & $3.78_{-0.47}^{+0.82}$ & $3.72 \pm 0.05$ \\
\hline A0548e & $2.93_{-0.15}^{+0.19}$ & $1.05 \pm 0.03$ & $1.49_{-0.17}^{+0.83}$ & $0.97_{-0.04}^{+0.03}$ & $0.18_{-0.02}^{+0.01}$ & $2.65_{-0.0}^{+0.47}$ & $4.17 \pm 0.08$ \\
\hline A0548w & $\left(1.68_{-0.53}^{+0.77}\right)$ & $0.19 \pm 0.02$ & $1.00_{-0.52}^{+0.58}$ & $0.85_{-0.19}^{+0.14}$ & $0.08_{-0.04}^{+0.07}$ & $0.82_{-0.43}^{+0.49}$ & - \\
\hline A0644 & $6.54_{-0.26}^{+0.27}$ & $8.35 \pm 0.15$ & $8.41_{-3.81}^{+2.52}$ & $1.68_{-0.031}^{+0.19}$ & $0.16_{-0.02}^{+0.04}$ & $13.85_{-297}^{+1.53}$ & $6.34 \pm 0.06$ \\
\hline A1413 & $6.56_{-0.44}^{+0.65}$ & $10.71 \pm 0.30$ & $9.77_{-4.58}^{+2.78}$ & $1.65_{-0.31}^{+0.31}$ & $0.17_{-0.03}^{+0.02}$ & $16.85_{-3.46}^{+1.94}$ & - \\
\hline A1689 & $8.58_{-0.40}^{+0.84}$ & $19.48 \pm 0.34$ & $14.98_{-8.38}^{+5.82}$ & $1.84_{-0.44}^{+0.21}$ & $0.16_{-0.03}^{+0.13}$ & $23.26_{-5.42}^{+3.69}$ & - \\
\hline A 1775 & $3.66_{-0.20}^{+0.30}$ & $3.09 \pm 0.09$ & $\begin{array}{l}4.19_{-1.69}^{+1.36} \\
4\end{array}$ & $1.32_{-0.21}^{+0.13}$ & $0.18_{-0.03}^{+0.03}$ & $7.55_{-2.07}^{+2.42}$ & - \\
\hline A 1800 & $\left(5.02_{-1.09}^{+2.20}\right)$ & $2.85 \pm 0.37$ & $5.94_{-3.59}^{-1.69}$ & $1.49_{-0.40}^{+0.21}$ & $0.13_{-0.09}^{+0.03}$ & $\begin{array}{r}7.63_{-4.28}^{+4.07} \\
\end{array}$ & - \\
\hline A1914 & $8.41_{-0.58}^{+0.60}$ & $17.04 \pm 0.38$ & $\begin{array}{r}-3.59 \\
11.84_{-5.84}^{+3.65}\end{array}$ & $1.72_{-0.35}^{+0.40}$ & $0.18_{-0.03}^{+0.09}$ & $21.20_{-4.72}^{+2.58}$ & - \\
\hline $\mathrm{A} 2151 \mathrm{w}$ & $2.58_{-020}^{+0.19}$ & $0.89 \pm 0.03$ & $1.60_{-0.61}^{+0.85}$ & $1.00_{-0.075}^{+0.07}$ & $0.14_{-0.02}^{-0.05}$ & $2.25_{-0.51}^{+0.44}$ & $3.44 \pm 0.14$ \\
\hline A2319 & $8.84_{-0.24}^{+0.20}$ & $16.37 \pm 0.26$ & $13.57_{-4.59}^{+2.15}$ & $1.99_{-0.26}^{+0.15}$ & $0.24_{-0.02}^{-0.02}$ & $32.26_{-5.92}^{+3.88}$ & $14.63 \pm 0.04$ \\
\hline A2734 & $5.07_{-0.02}^{+0.24}$ & $2.40 \pm 0.10$ & $4.82_{-1.56}^{+0.59}$ & $1.40_{-0.17}^{+0.08}$ & $0.14_{-0.02}^{+0.02}$ & $6.80_{-152}^{+0.9 .92}$ & $3.13 \pm 0.09$ \\
\hline A2877 & $\begin{array}{l}a .0 .42 \\
a_{3} .50_{-1.10}^{+2.20}\end{array}$ & $0.40 \pm 0.01$ & $6.88_{-379}^{+6.56}$ & $1.64_{-0.38}^{+0.12}$ & $0.04_{-0.02}^{+0.03}$ & $3.04_{-0.13}^{+0.52}$ & - \\
\hline A3395n & $5.11_{-0.43}^{+0.10}$ & $1.63 \pm 0.11$ & $\begin{array}{l}8.13_{-469}^{+5.79} \\
0.53\end{array}$ & $1.69_{-0.42}^{+0.38}$ & $\begin{array}{l}-0.02 \\
0.10_{-0.07}^{+0.12}\end{array}$ & $\begin{array}{r}-0.63 \\
8.24_{-5.34}^{+2.20}\end{array}$ & - \\
\hline A3528n & $\begin{array}{r}-0.43 \\
4.79_{-0.54}^{+0.50}\end{array}$ & $1.56 \pm 0.06$ & $\begin{array}{r}-4.69 \\
4.49_{-153}^{+0.78}\end{array}$ & $1.38_{-018}^{+0.08}$ & $\begin{array}{l}0.0 .07 \\
0.12_{-0.02}^{+0.04}\end{array}$ & $\begin{array}{l}0.533_{-1.54}^{+0.67} \\
5.37\end{array}$ & - \\
\hline A3528s & $4.60_{-0.27}^{+0.44}$ & $2.20 \pm 0.06$ & $2.76_{-0.14}^{+0.53}$ & $\begin{array}{l}1.07_{-0.07}^{-0.05} \\
1.18\end{array}$ & $0.20_{-0.02}^{+0.02}$ & $5.56_{-0.13}^{+0.51}$ & - \\
\hline A3530 & $4.05_{-0.30}^{+0.32}$ & $1.21 \pm 0.06$ & $4.34_{-1.07}^{+1.44}$ & $1.37_{-015}^{-0.01}$ & $0.12_{-0.05}^{+0.02}$ & $5.22^{+0.83}$ & - \\
\hline A3532 & $4.41^{+0.19}$ & $2.20 \pm 0.06$ & $6.63^{-1.1 .17}$ & $1.57^{+0.09}$ & $0.12^{+0.08}$ & $\begin{array}{l}-1.38 \\
8.11^{+0.68}\end{array}$ & - \\
\hline A 3560 & $\left(3.90_{-1.23}^{+1.81}\right)$ & $1.57 \pm 0.06$ & $2.77_{-1.26}^{+1.84}$ & $1.18_{-0.22}^{+0.22}$ & $0.17_{-0.04}^{+0.06}$ & $4.61_{-1.36}^{+1.38}$ & $6.50 \pm 0.27$ \\
\hline A3627 & $5.62_{-0.12}^{+0.23}$ & $3.59 \pm 0.18$ & $4.92_{-0.91}^{+0.67}$ & $1.48_{-010}^{+0.22}$ & $0.19^{+0.05}$ & $9.57^{+1.36}$ & - \\
\hline A3695 & $\left(6.76_{-214}^{+3.11}\right)$ & $5.89 \pm 0.89$ & $7.03^{+4.66}$ & $1.55_{-041}^{-0.10}$ & $0.18^{+0.048}$ & $12.82^{-2.66}$ & - \\
\hline A3822 & $5.12^{+0.43}$ & $4.83 \pm 0.58$ & $4.69^{-1.1 .19}$ & $1.37^{-0.11}$ & $0.19^{+0.12}$ & $9.07^{+2.53}$ & $5.38 \pm 0.10$ \\
\hline A3827 & $\left(7.66^{+3.34}\right)$ & $7.94 \pm 0.78$ & $15.50^{-1.48}+81$ & $2.01^{-0.46}$ & $0.13^{+0.08}$ & $19.88^{-411.11}$ & - \\
\hline A3888 & $\left(8.68^{+4.01}\right)$ & $10.09 \pm 0.39$ & $29.81^{-24.96}$ & $2.38^{+0.54}$ & $0.07^{-0.08}$ & $\begin{array}{r}-11.45 \\
21.97^{+3.60}\end{array}$ & - \\
\hline A3921 & $5.39^{+0.38}$ & $4.83 \pm 0.14$ & $6.59^{+1.50^{3}}$ & $1.51^{-0.11}$ & $0.16^{+0.03}$ & $10.76^{+6.41}$ & - \\
\hline AWM7 & $3.70_{-0.05}^{+0.35}$ & $2.10 \pm 0.07$ & $4.92_{-2.26}^{+2.32}$ & $1.48_{-02}^{+0.11}$ & $0.12_{-0.02}^{+0.03}$ & $5.85_{-110}^{+0.705}$ & $2.90 \pm 0.06$ \\
\hline HCG 94 & $\begin{array}{l}3.30_{-0.16}^{+0.17} \\
\text { (1) }\end{array}$ & $1.28 \pm 0.02$ & $2.25_{-0.60}^{+0.26}$ & $1.11_{-0.11}^{+0.27}$ & $0.15_{-0.01}^{+0.03}$ & $3.41_{-0.49}^{+0.10}$ & $2.51 \pm 0.11$ \\
\hline IIZw 108 & $\left(4.28_{-1.35}^{+0.168}\right)$ & $1.98 \pm 0.24$ & $3.85_{-2.00}^{+2.60}$ & $1.32_{-0.28}^{+0.25}$ & $0.15_{-0.07}^{+0.011}$ & $5.65_{-2.73}^{+2.89}$ & - \\
\hline M 49 & $1.33_{-0.03}^{+0.03}$ & $0.02 \pm 0.00$ & $0.67_{-0.47}^{+0.40}$ & $0.77_{-0.26}^{+0.13}$ & $0.01_{-0.00}^{+0.01}$ & $0.09_{-0.04}^{+0.02}$ & - \\
\hline NGC 499 & $0.66_{-0.03}^{+0.03}$ & $0.04 \pm 0.00$ & $0.33_{-0.23}^{+0.45}$ & $0.60_{-0.20}^{+0.12}$ & $0.03_{-0.01}^{+0.04}$ & $0.08_{-0.03}^{+0.02}$ & - \\
\hline NGC 5813 & $0.76_{-0.19}^{+0.19}$ & $0.02 \pm 0.00$ & $0.43_{-0.33}^{+0.45}$ & $0.66_{-0.26}^{+0.18}$ & $0.01_{-0.01}^{+0.01}$ & $0.06_{-0.04}^{+0.07}$ & - \\
\hline NGc 5846 & $0.64_{-0.03}^{+0.04}$ & $0.01 \pm 0.00$ & $0.18_{-0.12}^{+0.13}$ & $0.49_{-0.16}^{+0.08}$ & $0.03_{-0.01}^{+0.03}$ & $0.05_{-0.02}^{+0.04}$ & - \\
\hline OPHIUCHU & $10.25_{-0.36}^{+0.30}$ & $12.14 \pm 0.39$ & $38.76_{-24.11}^{+21.59}$ & $2.91_{-0.81}^{+0.46}$ & $0.07_{-0.02}^{+0.09}$ & $28.60_{-6.11}^{+2.89}$ & - \\
\hline PERSEUS & $6.42_{-0.06}^{+0.06}$ & $16.39 \pm 0.20$ & $6.08_{-285}^{+1.55}$ & $1.58_{-0.30}^{+0.812}$ & $0.30_{-0.03}^{+0.02}$ & $18.10^{+1.81}$ & $8.63 \pm 0.07$ \\
\hline PKS 0745 & $6.37_{-0.20}^{+0.20}$ & $27.13 \pm 0.42$ & $7.11_{-3.91}^{+2.85}$ & $1.54_{-0.36}^{+0.18}$ & $0.28_{-0.05}^{+0.18}$ & $19.74_{-5.45}^{+2.46}$ & - \\
\hline RXJ 2344 & $\left(5.52_{-1.74}^{+2.55}\right)$ & $3.59 \pm 0.08$ & $8.89_{-5.25}^{+5.41}$ & $1.70_{-0.44}^{+0.20}$ & $0.11_{-0.03}^{+0.030}$ & $9.47_{-2.190}^{+1.35}$ & - \\
\hline S405 & $\left(5.02_{-1.59}^{+2.34}\right)$ & $2.90 \pm 0.39$ & $4.62_{-2.88}^{+3.14}$ & $1.39_{-0.39}^{+0.24}$ & $0.16_{-0.10}^{+0.13}$ & $7.59_{-4.37}^{+3.79}$ & - \\
\hline S540 & $\left(3.09_{-0.98}^{+1.33}\right)$ & $0.89 \pm 0.07$ & $2.52_{-1.40}^{+1.85}$ & $1.16_{-0.27}^{+0.39}$ & $0.11_{-0.05}^{+0.08}$ & $2.68_{-1.123}^{+1.18}$ & - \\
\hline S636 & $2.06_{-0.06}^{+0.07}$ & $0.38 \pm 0.03$ & $1.56_{-0.17}^{+0.40}$ & $1.01_{-0.11}^{+0.09}$ & $0.10_{-0.04}^{+0.07}$ & $1.52_{-0.62}^{+0.35}$ & - \\
\hline TRIANGUL & $9.06_{-0.33}^{+0.06}$ & $12.43 \pm 0.15$ & $14.84_{-5.28}^{+2.49}$ & $2.07_{-0.28}^{+0.11}$ & $0.20_{-0.02}^{+0.04}$ & $29.14_{-4.92}^{+2.35}$ & $12.01 \pm 0.05$ \\
\hline UGC 03957 & $\left(3.21_{-1.02}^{+1.38}\right)$ & $0.98 \pm 0.10$ & $3.32_{-2.03}^{+2.28}$ & $1.28_{-0.34}^{+0.28}$ & $0.08_{-0.04}^{+0.02}$ & $2.67_{-1.47}^{+1.6^{2}}$ & - \\
\hline ZwCl 1742 & $\left(6.05_{-1.91}^{+2.80}\right)$ & $4.54 \pm 0.33$ & $10.11_{-6.30}^{+5.91}$ & $1.78_{-0.49}^{+0.30}$ & $0.11_{-0.03}^{+0.13}$ & $11.20_{-3.22}^{+3.42}$ & - \\
\hline
\end{tabular}

Note: The symbols in $T_{\mathrm{h}}$ have the same meanings as in Table 4. 
Y. Chen et al.: Statistics of X-ray observables of galaxy clusters, Online Material $p 7$

Table 6. Fits to relations of $r_{\mathrm{c}}$ and $M_{500}$.

\begin{tabular}{ccccc}
\hline \hline Relation & Number of clusters & $B$ & $A$ & Comments \\
\hline$r_{\mathrm{c}}-M_{500}$ & 106 & $1.18 \pm 0.08$ & $0.253 \pm 0.036$ & ALL \\
$r_{\mathrm{c}}-M_{500}$ & 52 & $0.90 \pm 0.06$ & $0.023 \pm 0.036$ & CCCs \\
$r_{\mathrm{c}}-M_{500}$ & 54 & $0.66 \pm 0.12$ & $0.452 \pm 0.029$ & NCCCs \\
\hline
\end{tabular}

Note: The relations are given in the form: $\log _{10}\left(\frac{r_{\mathrm{c}}}{100 \mathrm{kpc}}\right)=A+B \cdot \log _{10}\left(\frac{M_{500}}{5 \times 10^{14} M_{\odot}}\right)$.

Table 7. Summary of the fits to the scaling relations.

\begin{tabular}{|c|c|c|c|c|}
\hline Relation & Number of clusters & $\bar{B}$ & $\bar{A}$ & Comments \\
\hline$M_{500}-T_{\mathrm{h}}$ & 88 & $1.54 \pm 0.06$ & $-0.112 \pm 0.014$ & ALL with $T_{\mathrm{h}}$ \\
\hline$M_{500}-T_{\mathrm{h}}$ & 47 & $1.48 \pm 0.07$ & $-0.140 \pm 0.015$ & $\mathrm{CCCs}$ \\
\hline$M_{500}-T_{\mathrm{h}}$ & 41 & $1.57 \pm 0.17$ & $-0.088 \pm 0.031$ & NCCCs \\
\hline$M_{500}-T_{\mathrm{h}}$ & 72 & $1.30 \pm 0.18$ & $-0.069 \pm 0.025$ & $T_{\mathrm{h}}>3.0 \mathrm{keV}$ \\
\hline$M_{500}-T_{\mathrm{h}}$ & 16 & $0.91 \pm 0.31$ & $-0.46 \pm 0.13$ & $T_{\mathrm{h}}<3.0 \mathrm{keV}$ \\
\hline$M_{\mathrm{gas}, 500}-T_{\mathrm{h}}$ & 88 & $2.29 \pm 0.09$ & $-0.269 \pm 0.015$ & ALL with $T_{\mathrm{h}}$ \\
\hline$M_{\mathrm{gas}, 500}^{\mathrm{gas}}-T_{\mathrm{h}}$ & 47 & $2.38 \pm 0.10$ & $-0.251 \pm 0.017$ & $\mathrm{CCCs}$ \\
\hline$M_{\mathrm{gas}, 500}-T_{\mathrm{h}}$ & 41 & $2.04 \pm 0.14$ & $-0.258 \pm 0.025$ & NCCCs \\
\hline$M_{\mathrm{gas}, 500}-T_{\mathrm{h}}$ & 72 & $1.89 \pm 0.10$ & $-0.221 \pm 0.017$ & $T_{\mathrm{h}}>3.0 \mathrm{keV}$ \\
\hline$M_{\mathrm{gas}, 500}-T_{\mathrm{h}}$ & 35 & $1.98 \pm 0.11$ & $-0.219 \pm 0.019$ & $T_{\mathrm{h}}>3.0 \mathrm{keV}, \mathrm{CCCs}$ \\
\hline$M_{\mathrm{gas}, 500}-T_{\mathrm{h}}$ & 37 & $1.80 \pm 0.18$ & $-0.219 \pm 0.033$ & $T_{\mathrm{h}}>3.0 \mathrm{keV}, \mathrm{NCCCs}$ \\
\hline$M_{\mathrm{gas}, 500}-T_{\mathrm{m}}$ & 71 & $1.70 \pm 0.06$ & $-0.184 \pm 0.015$ & ALL with $T_{\mathrm{m}}$ \\
\hline$M_{\mathrm{gas}, 500}-T_{\mathrm{m}}$ & 38 & $1.73 \pm 0.07$ & $-0.166 \pm 0.022$ & $\mathrm{CCCs}$ \\
\hline$M_{\mathrm{gas}, 500}-T_{\mathrm{m}}$ & 33 & $1.71 \pm 0.11$ & $-0.207 \pm 0.025$ & NCCCs \\
\hline$L_{\mathrm{X}}-T_{\mathrm{h}}$ & 88 & $2.73 \pm 0.13$ & $0.363 \pm 0.027$ & ALL with $T_{\mathrm{h}}$ \\
\hline$L_{\mathrm{X}}-T_{\mathrm{h}}$ & 47 & $2.88 \pm 0.15$ & $0.492 \pm 0.031$ & $\mathrm{CCCs}$ \\
\hline$L_{\mathrm{X}}-T_{\mathrm{h}}$ & 41 & $2.74 \pm 0.17$ & $0.227 \pm 0.034$ & NCCCs \\
\hline$L_{\mathrm{X}}-T_{\mathrm{h}}$ & 72 & $2.88 \pm 0.19$ & $0.332 \pm 0.034$ & $T_{\mathrm{h}}>3.0 \mathrm{keV}$ \\
\hline$L_{\mathrm{X}}-T_{\mathrm{h}}$ & 35 & $3.08 \pm 0.24$ & $0.457 \pm 0.039$ & $>3.0 \mathrm{keV}, \mathrm{CCCs}$ \\
\hline$L_{\mathrm{X}}-T_{\mathrm{h}}$ & 37 & $2.78 \pm 0.22$ & $0.213 \pm 0.044$ & $T_{\mathrm{h}}>3.0 \mathrm{keV}, \mathrm{NCCCs}$ \\
\hline$L_{\mathrm{X}}-T_{\mathrm{m}}$ & 71 & $2.23 \pm 0.11$ & $0.458 \pm 0.032$ & ALL with $T_{\mathrm{m}}$ \\
\hline$L_{\mathrm{X}}-T_{\mathrm{m}}$ & 38 & $2.31 \pm 0.14$ & $0.597 \pm 0.040$ & $\mathrm{CCCs}$ \\
\hline$L_{\mathrm{X}}-T_{\mathrm{m}}$ & 33 & $2.33 \pm 0.09$ & $0.286 \pm 0.031$ & NCCCs \\
\hline$L_{X}-M_{500}$ & 106 & $1.82 \pm 0.13$ & $0.521 \pm 0.039$ & ALL \\
\hline$L_{\mathrm{x}}-M_{500}$ & 88 & $1.77 \pm 0.12$ & $0.562 \pm 0.041$ & ALL with $T_{\mathrm{b}}$ \\
\hline$L_{\mathrm{X}}-M_{500}$ & 47 & $1.94 \pm 0.15$ & $0.763 \pm 0.050$ & $\mathrm{CCCs}$ \\
\hline$L_{X}-M_{500}$ & 41 & $1.75 \pm 0.25$ & $0.381 \pm 0.062$ & NCCCs \\
\hline$L_{\mathrm{X}}-M_{500}$ & 72 & $2.23 \pm 0.38$ & $0.485 \pm 0.053$ & $T_{\mathrm{h}}>3.0 \mathrm{keV}$ \\
\hline$L_{X}-M_{500}$ & 35 & $3.05 \pm 0.60$ & $0.674 \pm 0.074$ & $3.0 \mathrm{keV}, \mathrm{CCCs}$ \\
\hline$L_{X}-M_{500}$ & 37 & $1.84 \pm 0.42$ & $0.350 \pm 0.087$ & $T_{\mathrm{h}}>3.0 \mathrm{keV}$, NCCCs \\
\hline$L_{\mathrm{nir}}-T_{\mathrm{h}}$ & 58 & $1.34 \pm 0.09$ & $0.586 \pm 0.017$ & ALL with $T_{\mathrm{m}}$ \\
\hline$L_{\mathrm{nir}}-T_{\mathrm{h}}$ & 31 & $1.17 \pm 0.11$ & $0.565 \pm 0.017$ & CCCs \\
\hline$L_{\mathrm{nir}}-T_{\mathrm{h}}$ & 27 & $1.47 \pm 0.15$ & $0.593 \pm 0.035$ & NCCCs \\
\hline$L_{\mathrm{nir}}-M_{\mathrm{gas}, 500}$ & 62 & $0.702 \pm 0.055$ & $0.741 \pm 0.016$ & ALL \\
\hline$L_{\text {nir }}-M_{\text {gas }, 500}$ & 33 & $0.604 \pm 0.062$ & $0.686 \pm 0.018$ & $\mathrm{CCCs}$ \\
\hline$L_{\mathrm{nir}}-M_{\mathrm{gas}, 500}^{\mathrm{gan}}$ & 29 & $0.756 \pm 0.080$ & $0.778 \pm 0.024$ & NCCCs \\
\hline
\end{tabular}

Note: The relations are given in the form: $\log _{10}\left(\frac{M_{500}}{5 \times 10^{14} M_{\odot}}\right)=A+B \cdot \log _{10}\left(\frac{T}{4 \mathrm{keV}}\right), \log _{10}\left(\frac{M_{\mathrm{gaa}, 500}}{10^{14} M_{\odot}}\right)=A+B \cdot \log _{10}\left(\frac{T}{4 \mathrm{keV}}\right), \log _{10}\left(\frac{L_{X}}{10^{44} \mathrm{erg} / \mathrm{s}}\right)=$ $A+B \cdot \log _{10}\left(\frac{T}{4 \mathrm{keV}}\right), \log _{10}\left(\frac{L_{\mathrm{X}}}{10^{44} \mathrm{erg} / \mathrm{s}}\right)=A+B \cdot \log _{10}\left(\frac{M_{500}}{5 \times 10^{14} M_{\odot}}\right), \log _{10}\left(L_{\mathrm{nir}}\right)=A+B \cdot \log _{10}\left(\frac{T}{4 \mathrm{keV}}\right) \operatorname{and}_{\log _{10}}\left(L_{\mathrm{nir}}\right)=A+B \cdot \log _{10}\left(\frac{M_{\mathrm{gas}, 500}}{10^{14} M_{\odot}}\right)$. 